\title{
Review of Multiphase Flow and Pollutant Transport Models for the Hanford Site
}

\author{
C. T. Kincaid \\ P. J. Mitchell
}

November 1986

Prepared for the U.S. Department of Energy under Contract DE-AC06-76RLO 1830

Pacific Northwest Laboratory Operated for the U.S. Department of Energy by Battelle Memorial Institute 


\title{
DISCLAIMER
}

This report was prepared as an account of work sponsored by an agency of the United States Government. Neither the United States Government nor any agency thereof, nor Battelle Memorial Institute, nor any of their employees, makes any warranty, expressed or implied, or assumes any legal liability or responsibility for the accuracy, completeness, or usefulness of any information, apparatus, product, or process disclosed, or represents that its use would not infringe privately owned rights. Reference herein to any specific commercial product, process, or service by trade name, trademark, manufacturer, or otherwise, does not necessarily constitute or imply its endorsement, recommendation, or favoring by the United States Government of any agency thereof, or Battelle Memorial Institute. The views and opinions of authors expressed herein do not necessarly state or reflect those of the United States Government or any agency thereof, or Battelle Memorial Institute.

\author{
PACIFIC NORTHWEST LABORATORY \\ operated by \\ BATTELLE \\ for the \\ UNITED STATES DEPARTMENT OF ENERGY \\ under Contract DE-ACO6-76RLO 1830
}

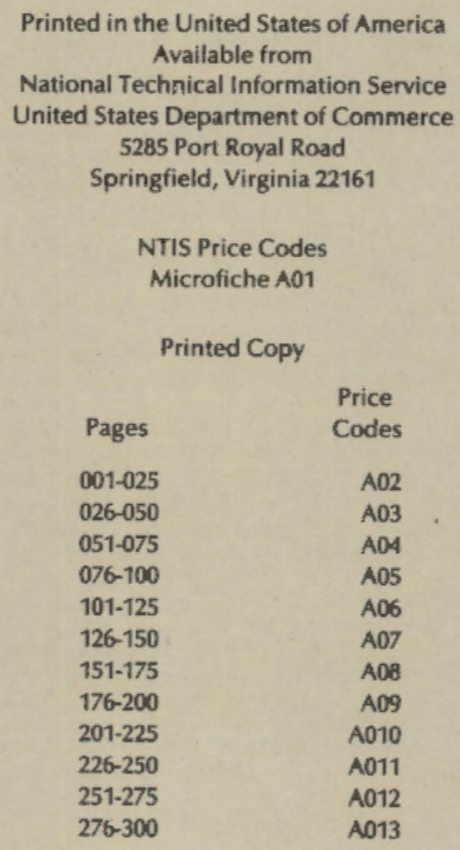


REVIEW OF MULTIPHASE FLOW AND POLLUTANT TRANSPORT MODELS FOR THE HANFORD SITE
C. T. Kincaid
P. J. Mitchell

November 1986

Prepared for the U.S. Departinent of Energy under Contract DE-ACO6-76RLO 1830

Pacific Northwest Laboratory Richland, Washington 99352 
PREFACE

As a result of operations at the Hanford site, wastes have been stored and disposed of in the ground. In some cases, pollutants from these wastes have reached the ground water, while in other cases the potential for ground-water contamination depends on the rate of migration through partially saturated soil deposits above the water table. The distribution of pollutants, such as inorganic and organic chemicals have generally not been investigated at the Hanford site.

This report provides a review of the physical processes, geochemical reactions, and microbiological kinetics that interact to determine the migration and fate of these pollutants. This review of processes and reactions provides a background from which codes for the analysis of contaminant migration and fate can be evaluated. Single codes representing classes of pollutant migration problems are cited to show how commonly employed and publicly available codes are not always applicable to the complex problems of multiphase fluid flow and pollutant migration. This review provides guidance on selecting and using codes; it also provides recommendations for development work needed to address deficiencies identified in existing models, codes, and data bases.

When applied in a site-specific setting, the analytical and numerical models discussed in this document aid in site characterization and monitoring network design by pointing out areas where more data are needed. Site-specific models can also be used in conjunction with monitoring to assess compliance with applicable regulations and to provide important information during the evaluation, selection, design, and implementation of restoration and protection strategies. Codes calibrated to establish a site-specific model can be applied to simulate subsurface migration patterns for waste constituents from existing or changing disposal practices or accidental spills; however, estabiished codes are virtually limited to the simulation of miscible solute in a single-fluid phase.

This report was written under a contract for the Richland Operations Office of the U.S. Department of Energy, as part of the Ground-Water Monitoring Project for the Hanford site. 


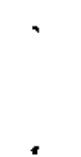

. 


\section{SUMMARY}

Pacific Northwest Laboratory (PNL) reviewed process models and computer codes available for modeling multiphase ground-water flow and solute transport for site characterization and ground-water monitoring network design at the Hanford site. The objectives of the work were to review available models and codes for simulating the movement of pollutants through the vadose zone and ground-water system; give guidance in code selection and use; and make recommendations for development work to address, model, and code deficiencies. The scope of work for this model and code evaluation was limited to the review of published code evaluations and summaries.

It was found that four approaches were used to model the transport of solute in a single-fluid phase or multiphase fluid environment. These approaches developed primarily for transport in a single-fluid phase included 1) the classical advection-dispersion model, 2) the kinematic pathline model, 3) the mobile-immobile model using the advection-dispersion approach, and 4) the stochastically based models. The variety of available approaches suggests that unresolved questions remain regarding transport phenomena in singlefluid phase systems.

This report includes several recommendations. First, a professional geohydrologist with modeling experience should be employed to perform all sitespecific modeling. The geohydrologist will, in cooperation with site managers, define the study objectives, collect site data, develop the conceptual model, identify important processes at the site, and select the code(s) to be used. Because code selection is site specific, varies with study objectives, and is often an iterative process, no single code includes all the processes necessary to simulate any waste disposal site. Hundreds of ground-water flow and solute transport codes are availabie and in use. The geohydrologist must technically evaluate the selected code before its installation and use as a site-specific model to ensure that it includes the relevant processes and functions claimed in its documentation. 
Second, studies should be conducted to identify parameters to be measured in the field that correspond to the input parameters required by the various solute transport models. The classical advection-dispersion model moves the solute by water-coincident advection. Heterogeneities of the porous media cause dispersion or deviations from the mean water velocity. Questions regarding the validity of this model have arisen out of a lack of measurable field-scale parameters that correspond to dispersion parameters used as model input.

Third, although the data required for mechanistic-source release modeling are rarely available, it must be noted that the estimated rate and duration of release can greatly affect the transport simulation. Thus, mechanistic releas? models should be used whenever sufficient data exist. Release models based on simplified physical, geochemical, and microbiological models should be used only when information ahout specific release mechanisms is absent. Note that fraction-remaining models can be used either for the simplified or the mechanistic models to interface between the source release and solute transport models.

Finally, it is recommended that knowledge of processes and reactions of single-phase and multiphase flow and solute transport, and their mathematics be advanced to address multiphase transport issues. Stochastic approaches to modeling transport phenomena should be developed to address the dispersion process and relate it to measurable parameters. It is also recommended that methods re developed to predict the expected value and uncertainty in chemical migration. Investment in an advanced capability to predict multidimensional and multiphase system behavior must strike a balance between providing an expedient tool for the manager and providing an all-encompassing physicochemical model of multiphase systems. 
CONTENTS

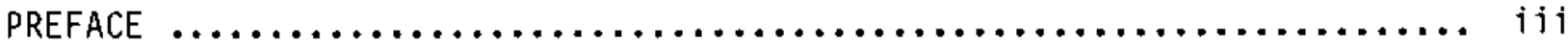

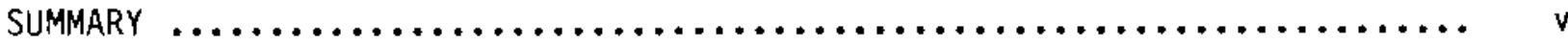

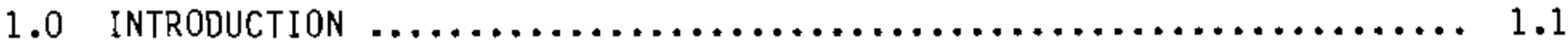

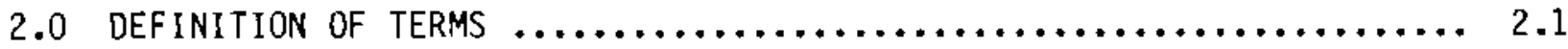

3.0 PHYSICAL PROCESSES ANO CHEMICAL REACTIONS $\ldots \ldots \ldots \ldots \ldots \ldots \ldots \ldots \ldots . .1$

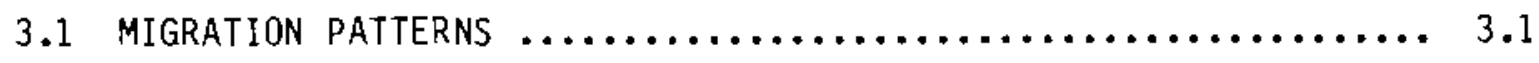

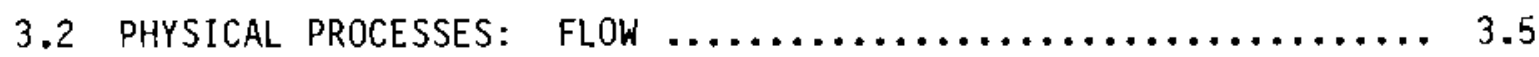

3.2 .1 Single-Phase Flow $\ldots \ldots \ldots \ldots \ldots \ldots \ldots \ldots \ldots \ldots \ldots \ldots, 3.6$

3.2 .2 Multiphase Flow $\ldots \ldots \ldots \ldots \ldots \ldots \ldots \ldots \ldots \ldots \ldots, 3.7$

3.2.3 Physical Parameters $\ldots \ldots \ldots \ldots \ldots \ldots \ldots \ldots \ldots \ldots \ldots \ldots \ldots \ldots \ldots \ldots, 3.9$

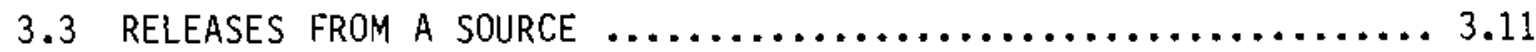

3.3.1 Isolation Afforded by Containment $\ldots \ldots \ldots \ldots \ldots \ldots \ldots . . .11$

3.3.2 Simple Models of Source Release $\ldots \ldots \ldots \ldots \ldots \ldots \ldots \ldots . .12$

3.3.3 Mechanistic Models of Source Release $\ldots \ldots \ldots \ldots \ldots \ldots \ldots .13$

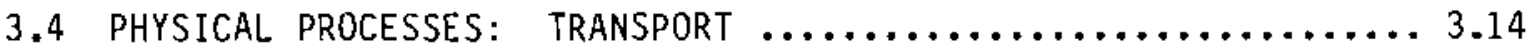

3.4.1 Advection/Convection $\ldots \ldots \ldots \ldots \ldots \ldots \ldots \ldots \ldots \ldots \ldots \ldots, 14$

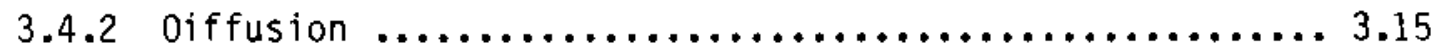

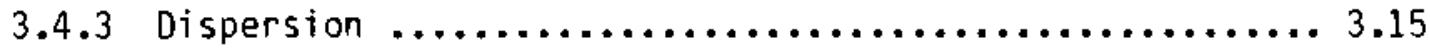

3.5 CHEMICAL REACTIONS: GEOCHEMISTRY, DECAY, AND MICROBIOLOGY $\ldots \ldots 3.17$

3.5.1 Adsorption/Desorption $\ldots \ldots \ldots \ldots \ldots \ldots \ldots \ldots \ldots \ldots \ldots \ldots \ldots \ldots \ldots \ldots, 18$

3.5.2 Precipitation/Dissolution $\ldots \ldots \ldots \ldots \ldots \ldots \ldots \ldots \ldots \ldots \ldots \ldots \ldots, 3.20$

3.5.3 Chemical Speciation $\ldots \ldots \ldots \ldots \ldots \ldots \ldots \ldots \ldots \ldots \ldots \ldots \ldots \ldots \ldots \ldots \ldots \ldots, 21$

3.5.4 0xidation and Reduction $\ldots \ldots \ldots \ldots \ldots \ldots \ldots \ldots, \ldots, \ldots \ldots \ldots, 21$

3.5 .5 Radioactive Decay $\ldots \ldots \ldots \ldots \ldots \ldots \ldots \ldots \ldots \ldots \ldots \ldots \ldots \ldots \ldots \ldots \ldots \ldots \ldots \ldots, 21$ 
3.5.6 Microbiological Degradation $\ldots \ldots \ldots \ldots \ldots \ldots \ldots \ldots \ldots \ldots \ldots \ldots \ldots \ldots .22$

3.5 .7 Fractured Media $\ldots \ldots \ldots \ldots \ldots \ldots \ldots \ldots \ldots \ldots \ldots \ldots \ldots \ldots \ldots \ldots \ldots \ldots \ldots \ldots, 22$

3.6 UNCERTAINTY ANALYSES $\ldots \ldots \ldots \ldots \ldots \ldots \ldots \ldots \ldots \ldots \ldots \ldots \ldots \ldots \ldots \ldots \ldots \ldots \ldots \ldots, 24$

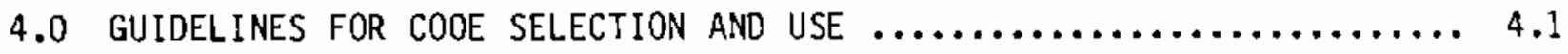

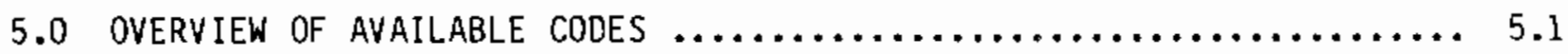

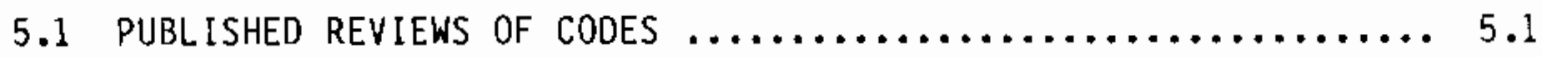

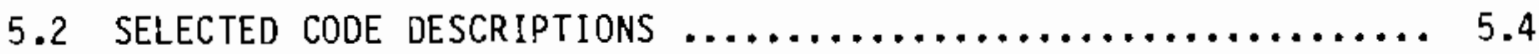

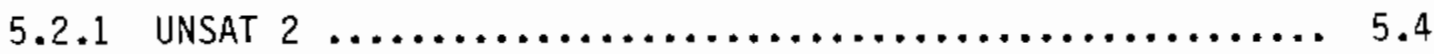

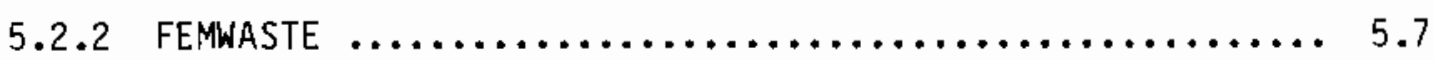

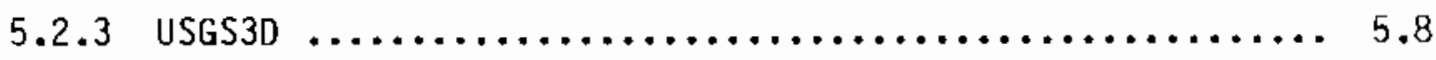

$5.2 .4 \operatorname{MOC} \ldots \ldots \ldots \ldots \ldots \ldots \ldots \ldots \ldots \ldots \ldots \ldots \ldots \ldots \ldots \ldots \ldots \ldots, 5.9$

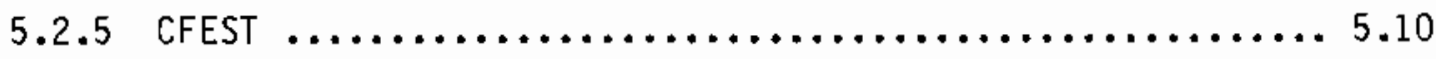

5.3 APPLICABILITY OF AVAILABLE CODES $\ldots \ldots \ldots \ldots \ldots \ldots \ldots \ldots \ldots \ldots . . \ldots \ldots \ldots$

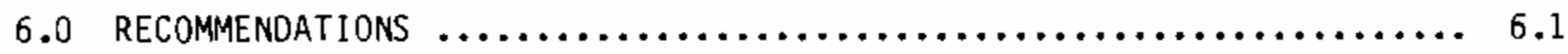

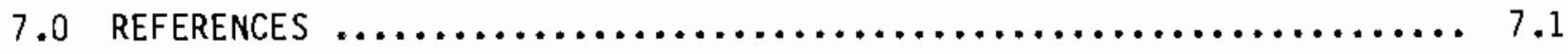




\section{FIGURES}

3.1 Schematic Diagram of a Chemical Spill of a Volume Less than the Retention Capacity of the Partially-Saturated Soil Profile ........ 3.2

3.2 Schematic Diagram of a Lighter-than-Water Chemical Spilled in a Volume Greater than the Retention Capacity of the Soil ........... 3.3

3.3 Schematic Diagram of a Heavier-than-Water Chemical Spilled in a Volume Greater than the Retention Capacity of the Soil ........... 3.4

3.4 Schematic Diagram of the Migration of a Chemical Vapor with a Density of Greater than One, Relative to Soil-Air ............... 3.5

3.5 Relative Permeabilities for a Wetting and a Nonwetting Fluid as a Function of the Degree of Saturation $\ldots \ldots \ldots \ldots \ldots \ldots \ldots . . .7$

3.6 Relative Permeabilities for Three-Phase Flow ................ 3.9

4.1 Systems Model Components $\ldots \ldots \ldots \ldots \ldots \ldots \ldots \ldots \ldots \ldots \ldots \ldots \ldots \ldots \ldots . \ldots \ldots$

\section{TABLES}

5.1 Number of Codes Available for Modeling Various Flow and Transport Processes ................................. 5.2

5.2 Summary of Capabilities for Selected Codes $\ldots \ldots \ldots \ldots \ldots \ldots \ldots \ldots \ldots$ 


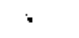




\subsection{INTRODUCT ION}

A review of computer codes available for modeling multiphase ground-water flow and solute transport was conducted by the Pacific Northwest Laboratory (PNL) for the U.S. Department of Energy (DOE). The need for such a modeling capability was prompted by the growing concern about the presence and movement of hazardous, organic chemicals in the ground water. These chemicals may exist in the subsurface as either a separate, immiscible fluid mingling with the ground water or a dilute, miscible solute mixed into the ground water.

This report discusses state-of-the-art deterministic modeling of groundwater flow and contaminant transport available to the DOE-contractor community. Codes that may be useful in modeling the important processes of hazardous chemical movement are summarized. Terms used in this document are defined in Section 2.0. Section 3.0 summarizes physical processes and chemical reactions important to the migration and attenuation of solutes. This summary does not include mathematicai statements of the governing relationships. Instead, conceptual definitions are included to provide the background needed to review the available simulation techniques. The last subsection in Section 3.0 summarizes the state of the art in uncertainty analyses. Section 4.0 contains guidelines for code selection and a modeling philosophy for code use. Following the guidelines, the capabilities of selected codes as described in the literature are summarized in a matrix format and in narrative form in Section 5.0. Recommendations for overall improvements in modeling capabilities, are discussed in Section 6.0. These improvements are based on the differences that exist between the operative physical and chemical models and the models incorporated in existing codes. 
、

. 


\subsection{DEF INITION OF TERMS}

Fluids that migrate in the subsurface environment can be grouped with regard to their migration behavior as either miscible (mixes) with water or immiscible (does not mix) with water. Miscible fluids form a single phase, while immiscible fluids form two or more fluid phases (a fluid is either a liquid or a gas). Such a grouping of fluids is essential for discussion purposes because the movement of two or more immiscible fluids is distinctly different from the simultaneous movement of miscible fluids. The flow of immiscible fluids gives rise to two-phase or multiphase flow and transport; miscible fluids give rise to single-phase flow and transport. Single-phase and multiphase flow are described in more detail in Section 3.2.

Models are distinguished here from codes. The former are the descriptions in either words or equations of physical processes and chemical reactions; the latter are sets of logic statements executable on a computer. The models believed to describe or influence solute transport in subsurface media are discussed throughout Section 3.0 . 


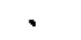




\subsection{PHYSICAL PROCESSES AND CHEMICAL REACTIONS}

In this section, the relevant processes and reactions are described to provide the necessary background from which to judge the adequacy or completeness of existing codes. After a discussion of the various migration patterns possible for miscible or immiscible fluids, the processes and reactions germane to flow, releases from a source, transport, and geochemistry are described in this section. This order (i.e., flow, releases, transport, and geochemistry) corresponds to the successively more complex problems described for 1) watermovement, 2) source releases, 3) transport mechanisms for inert or conservative tracers, and 4) geochemical reactions that lead to the attenuation of chemicals. The final subsection discusses the methods available for estimating the uncertainty associated with transport model predictions.

\subsection{MIGRATION PATTERNS}

Migration patterns associated with immiscible fluids are schematically described by Schville (1984). The extent and character of migration depends on the chemical characteristics, the spill volume, the area covered by the spill, the infiltration rate, and the retention capacity of the porous medium. The chemical characteristics of both the spilled chemical and the subsurface environment can play a role in determining whether the chemical will be a solid, a liquid, or a gas in the environment. The spill volume, area covered, and infiltration rate indicate the chemical quantities of concern. Retention capacity is a measure of the volume of immiscible liquid that can be held in the porous medium without appreciable movement. This volume is analogous to the volume of water prevented by the capillary force from draining because of the gravity force.

When the retention capacity of the partially saturated soil column is not exceeded, the bulk of the liquid chemical contaminant will be retained in the soil column. Migration of the contaminant to the far-field environment will occur as a result of its dissolution in water; it may also move in a distinct vapor phase, as will be discussed later. This contaminant will be conducted in the soil water at concentrations dictated by solubility, precipitation/ 
dissolution, and adsorption/desorption phenomena. Contaminated soil water arriving at the water table will be carried downgradient in the unconfined aquifer and in the capillary fringe. Figure 3.1 shows the ability of heterogeneous sediments within the partially saturated zone to laterally spread or broaden the contaminant plume with increasing depth. To estimate the retention capacity of the partially saturated soil column, the soil profile and moisture content must be known.

When the volume of chemical spill exceeds the retention capacity of the partially saturated soil profile, the chemical will reach the water table in its liquid phase. Chemicals that are less dense than and immiscible in water will remain in the capillary fringe of the partially saturated zone and near the water table in the saturated zone, as indicated in Figure 3.2. Immediately beneath the spill chemicals can be forced below the water table and into the saturated zone by the pressure of the overlying liquid chemical mound (e.g., analogous to ground-water mound created by water disposal). As the plume migrates downgradient, the overlying pressure decreases and buoyant forces

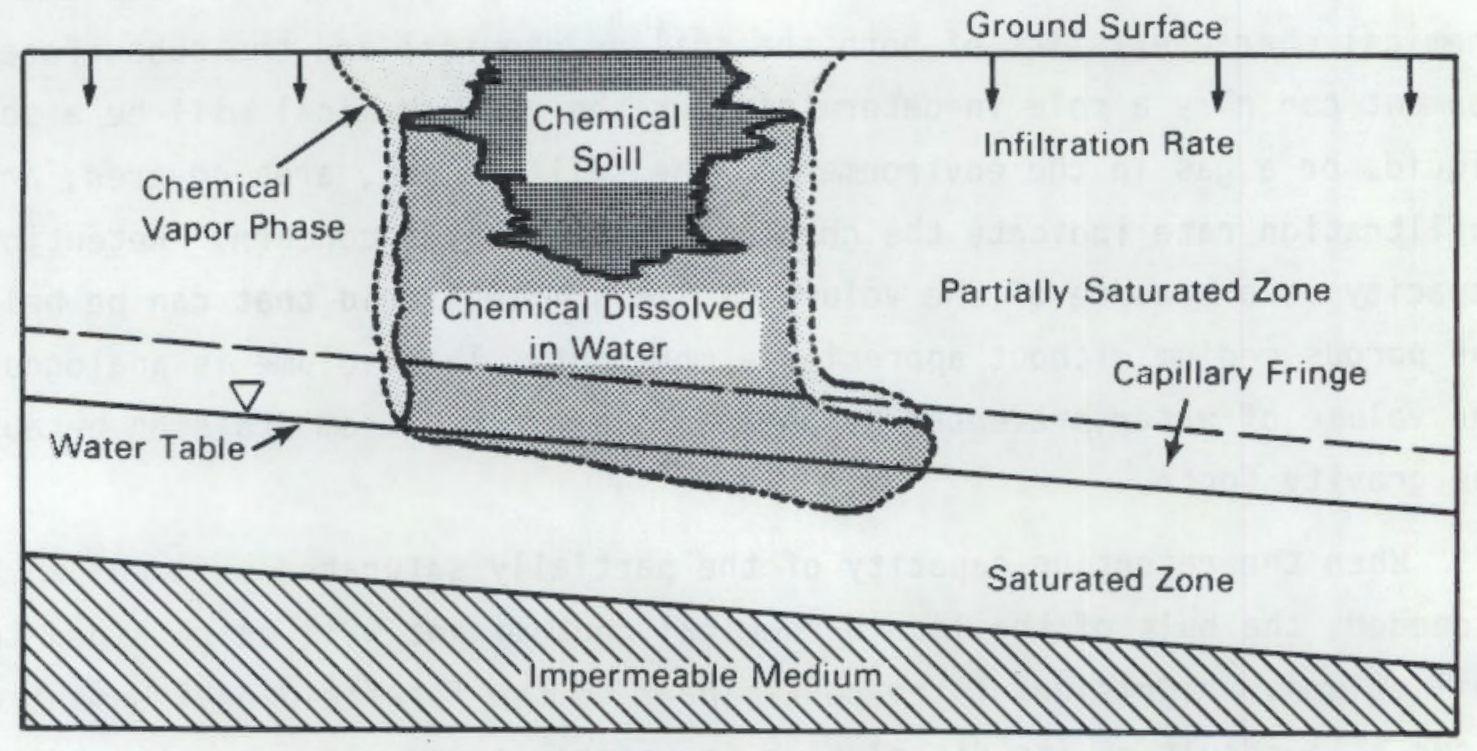

FIGURE 3.1. Schematic Diagram of a Chemical Spill of a Volume Less than the Retention Capacity of the Partially Saturated Soil Profile (After Schville 1984) 


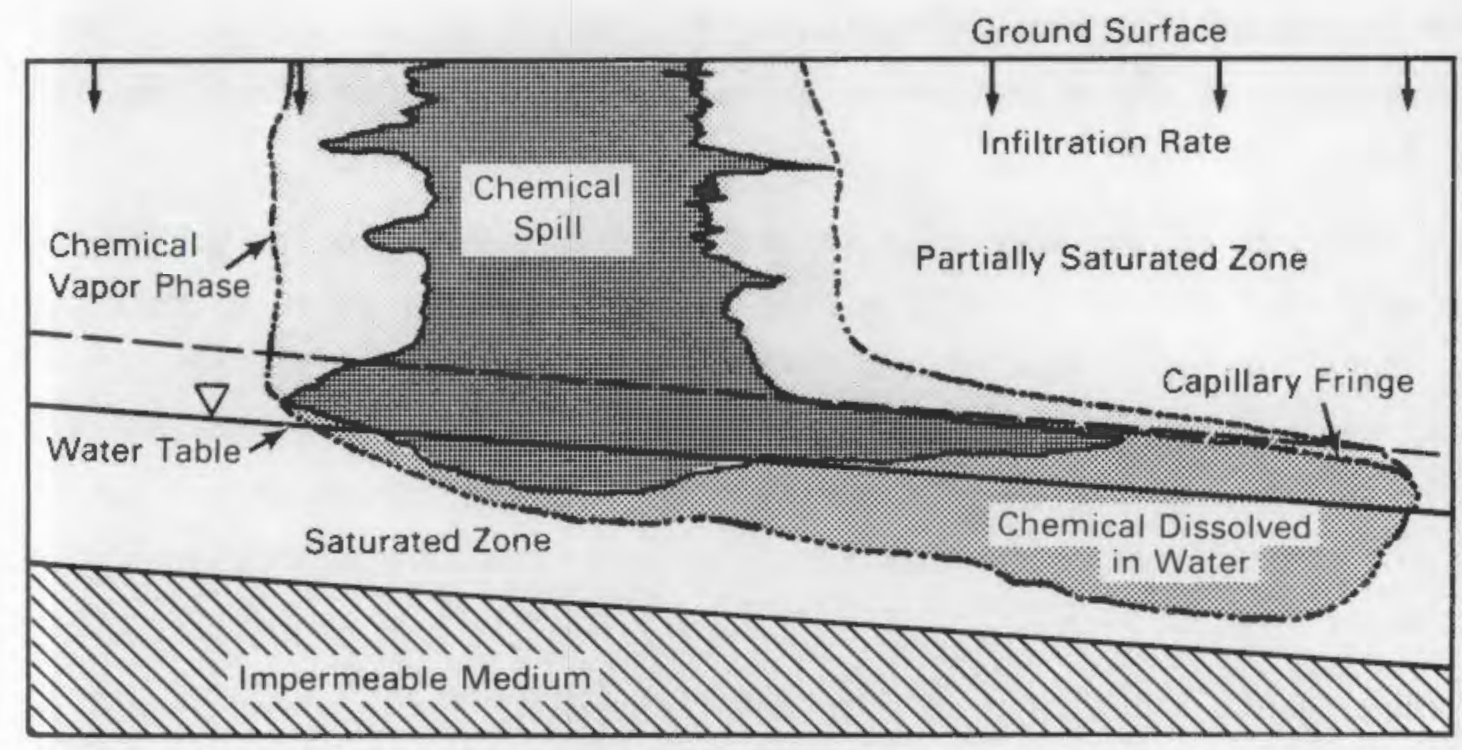

FIGURE 3.2. Schematic Diagram of a Lighter-than-Water Chemical Spill of a Volume Greater than the Retention Capacity of the Soil (After Schville 1984)

bring the lighter-than-water chemical up to the water table. The contamination will spread as a distinct liquid chemical phase and as a dissolved constituent in the ground water. Contamination could also spread as a distinct chemical vapor phase. Certainly, some fraction of the chemical will be held in the porous medium by the retention capacity mechanism. Release of this fraction, as a dissolved constituent in soil water and ground water, will be a long-term process.

A substantial spill of a heavier-than-water chemical, which is immiscible in water, will reach the water table and migrate toward the bottom of the aquifer. After reaching the water table, the chemical will continue to move principally in the downward direction by displacing the ground water. If the volume of the chemical moving into the saturated zone is greater than the retention capacity of the unconfined aquifer formation, the chemical will move through the entire saturated thickness of the unconfined aquifer. Depending on the physical/chemical properties of the chemical with respect to the impermeable formation, the chemical may continue its downward migration or form a mound above the impermeable bottom of the aquifer. Chemicals lying on the 
aquifer bottom will migrate by following the relief of the bedrock. These various aspects of the migration of a heavier-than-water chemical are shown in Figure 3.3.

As occurred in the partially saturated zone, heterogeneity within the saturated zone will act to spread the contaminant laterally as it migrates vertically. Note that the slope of the bottom topography (i.e., relief of the bedrock) may not coincide with the ground-water gradient; the chemical is driven by its own gradient not the hydraulic gradient of the ground water, and, hence, the chemical migration may actually move in a direction opposite to ground-water flow.

Many organic fluids are able to enter the vapor phase under normal subsurface conditions. Vaporization will be a function of the volatility of the chemical, which is mainly dependent on the vapor pressure and latent heat of evaporation of the fluid. It is possible that a distinct vapor phase contaminant plume will be created by such a mechanism. Certainly, the chemical may also contaminate the existing soil air. The density of vapor relative to soil

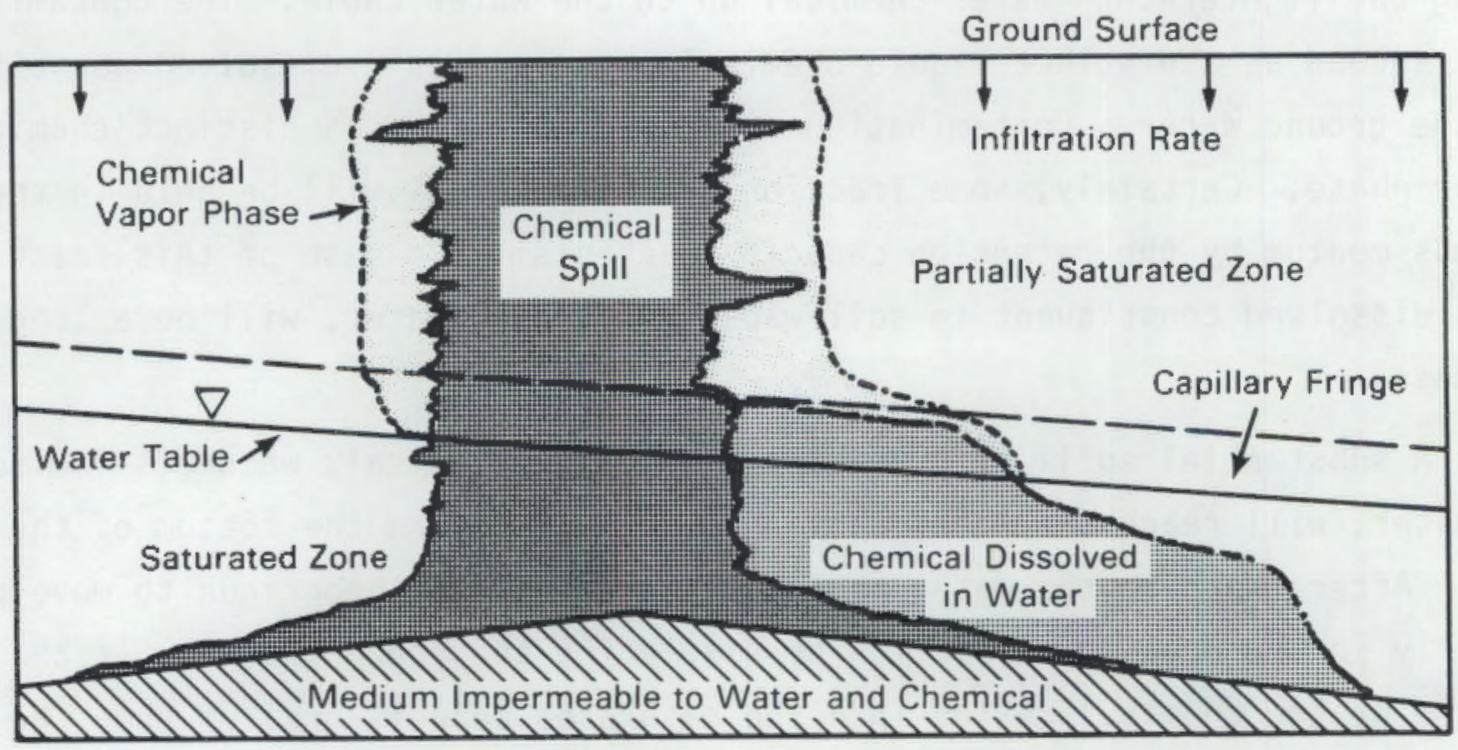

FIGURE 3.3. Schematic Diagram of a Heavier-than-Water Chemical Spill of a Volume Greater than the Retention Capacity of the Soil (After Schville 1984) 
air will determine whether the vapor phase plume will rise and escape to the atmosphere or sink in the partially saturated zone and spread above the capillary fringe. The latter case is depicted in Figure 3.4 .

\subsection{PHYSICAL PROCESSES: FLOW}

When the contaminant appears as a dissolved constituent in the soil water or ground water, water movement or flow will be the principal mechanism for transport. The migration scenarios described above reveal that contamination can migrate in the environment as a distinct liquid phase, a distinct gas phase, or as a dissolved contaminant in the soil water, soil air, or ground water. When the contaminant appears as a distinct liquid or gas phase, its movement is governed by the same physical principles that govern the movement of water or water vapor. However, when the contaminant appears as a distinct phase it competes with the water for the void space.

Models and codes of organic chemical migration are commonly categorized as 1) those for which fluid physics of immiscible organic liquids are emphasized, and 2) those for which organics appear as miscible constituents where in

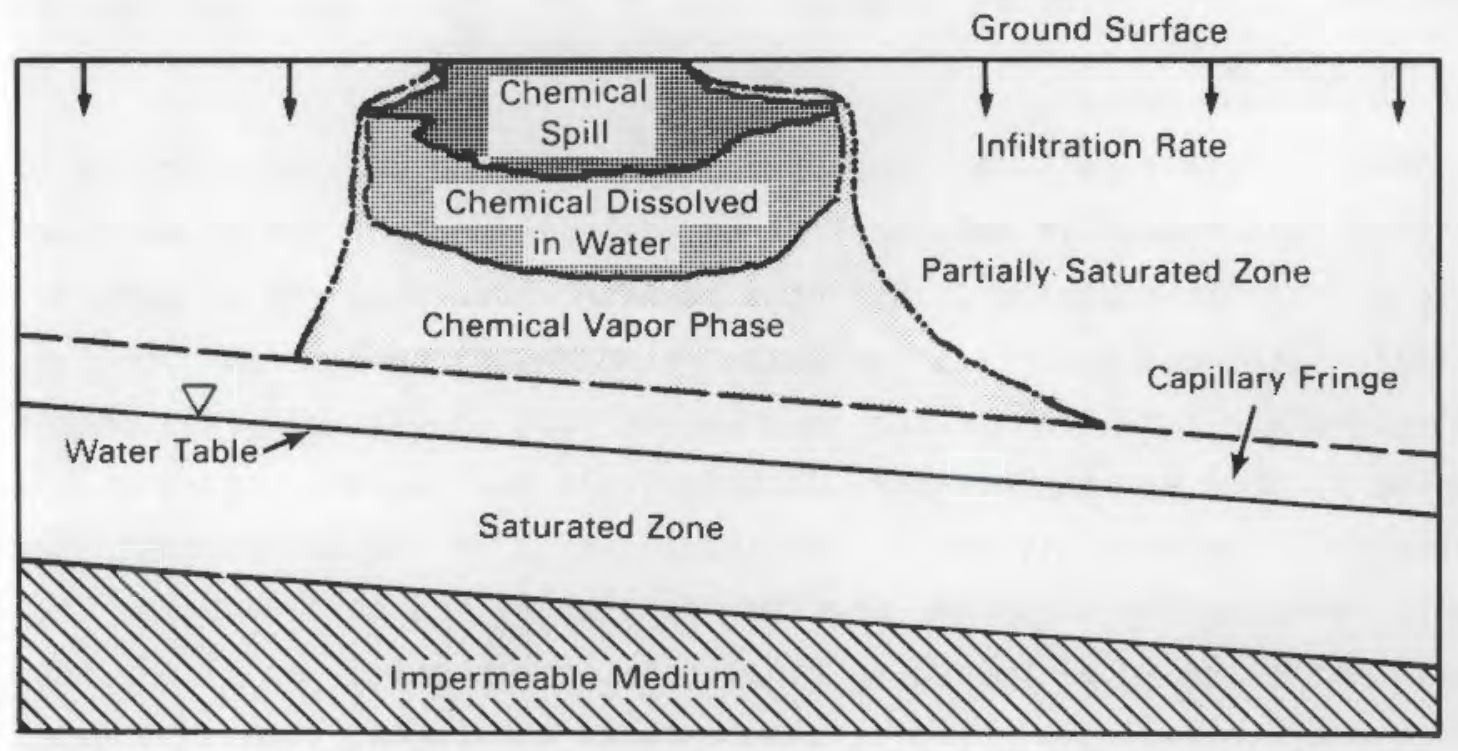

FIGURE 3.4. Schematic Diagram of the Migration of a Chemical Vapor with a Density of Greater than One, Relative to Soil-Air (After Schville 1984) 
chemical/microbiological reactions for dilute levels of contamination are emphasized. Existing models and codes can be used to model selected phases to the extent that vapor phase exchange and transport, geochemical reactions, and microbiological degradation can be incorporated in existing codes (i.e., insofar as the mathematical equations are unchanged by the addition of these processes and reactions). However, a broadly applicable multiphase modeling capability will not evolve from such serendipity because estimates of system performance would be based on one- and two-phase approximations.

Petroleum industry models and codes do not appear to be readily applicable to organic transport analyses. These codes address only fluid flow phenomena and neglect entirely transport and attenuation phenomena. Petroleum industry codes may only be useful in regard to the theory and methods they embody for simulating multiphase, immiscible-fluid flow.

The dimensionality of the physical problem must be matched by the dimensionality of the model. One- and two-dimensional characterizations of the subsurface are no longer widely accepted, especially by the legal systems if not the regulatory agencies. Actual flow and transport in the three-dimensional environment can differ markedly from predictions obtained from one- and twodimensional models based on idealizations of the three-dimensional world.

\subsubsection{Single-Phase Flow}

Single-phase flow is an important process in our understanding of chemical migration even though it only applies to the transport of dissolved chemicals in the ground-water aquifer. Strictly speaking, the movement of water in a partially saturated zone is a two-phase (1iquid-gas) phenomena. The importance of single-phase flow lies in the observation that 1) virtually all chemicals dissolve to some extent in water, 2) relatively low concentrations of some chemicals can be harmful, and 3 ) the leading edge of the contaminant plume will in most cases be the dissolved chemical carried by the soil water or ground water. While the total impact or final fate of a chemical spill may not be predicted by an analysis based on single-phase phenomena, such an analysis can identify arrival times of concern and initial levels of contamination. 
Water movement in a saturated and partially saturated porous medium is described by combining mass and momentum balance concepts. Mass balance implies a fluid mass balance, commonly referred to as the continuity equation. Momentum balance implies Darcy's law for fluid flow in a porous medium that relates volumetric flux to the gradient of pressure and the hydraulic conductivity (Freeze and Cherry 1979). In a saturated medium, the moisture content is at a maximum value equal to the effective porosity, and the hydraulic conductivity has a single value. Analysis of water movement in the saturated zone is greatly simplified by these constant parameters.

\subsubsection{Multiphase Flow}

The existence of distinct fluid phases competing for the same pore space gives rise to two-phase or multiphase flow. This competition is governed by mass and momentum balance equations and data that uniquely specify the balance between the fluids in the soil environment. The principle of simultaneous flow of two fluids is shown in the relative permeability versus the saturation diagram (Figure 3.5). The wetting fluid is usually water; examples of a nonwetting fluid are mineral oil, chlorohydrocarbons, and soil air. Flow of each fluid is proportional to its potential gradient, the permeability of the medium, the fluid density and viscosity, and the portion of pore space (i.e., cross-sectional area) that the fluid occupies.

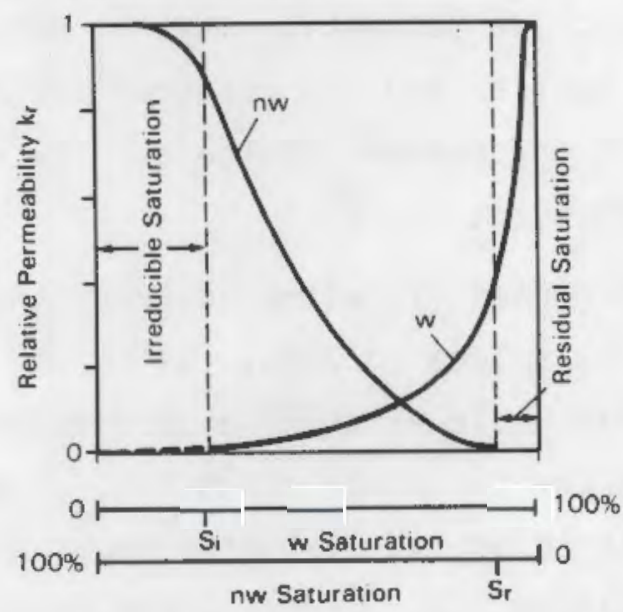

FIGURE 3.5. Relative Permeabilities for a Wetting (w) and a Nonwetting Fluid ( $\mathrm{nw}$ ) as a Function of the Degree of Saturation (Yaron et al. 1984) 
A fluid mass balance and Darcy's equation can be written for each of the fluids. When the detailed-flow phenomena in each fluid phase are of interest, as is the case with two liquids, the mass and momentum balance equations for each fluid should be solved. Consistent sets of saturation and potential for each fluid are obtained from such an analysis. However, when flow phenomena for only one of the two fluid phases are of interest, as is commonly the case with moisture movement in the partially saturated zone, the saturation and potential of the fluid of interest should be solved. The saturation of the second fluid can then be simply calculated given the porosity of the medium (i.e., given that it occupies the remaining pore space).

The relative permeability of the wetting and nonwetting fluids depends strongly on the degree of saturation (Figure 3.5). The curves for the two fluids show the typical nonlinear behavior of a fluid in a partially saturated environment. Note that the curve shown is a schematic diagram; unique curves exist for different fluids and media. In general, each fluid must reach a minimum saturation before it will flow. In the case of the wetting fluid (e.g., water), the minimum saturation is called the irreducible saturation. In the case of moisture movement in the vadose zone, soil physicists have found that irreducible saturation is actually a function of the suction pressure applied and the length of time one is willing to wait for the soil column to respond. Thus, irreducible saturation may not necessarily be single-valued. The nonwetting fluid must exceed its residual saturation before it will flow. Residual saturation is the measure of the ability of a soil to retain the bulk chemical spil1 (Figure 3.1).

One of the more complex migration patterns that may occur involves three phases in the partially saturated zone. Water and chemical would exist as liquid phases and soil air would exist as a gaseous phase. The flow process is more complicated than the two phase situation, although the same principles of mass and momentum balance apply. Relative permeabilities for a three-phase system are shown in Figure 3.6. Clearly, the individual fluids are immobile over relatively large areas of the diagram. Of interest is the relatively small region over which all three phases are simultaneously mobile, e.g., the region defined by relative permeabilities greater than $1 \%$ for all fluids. 


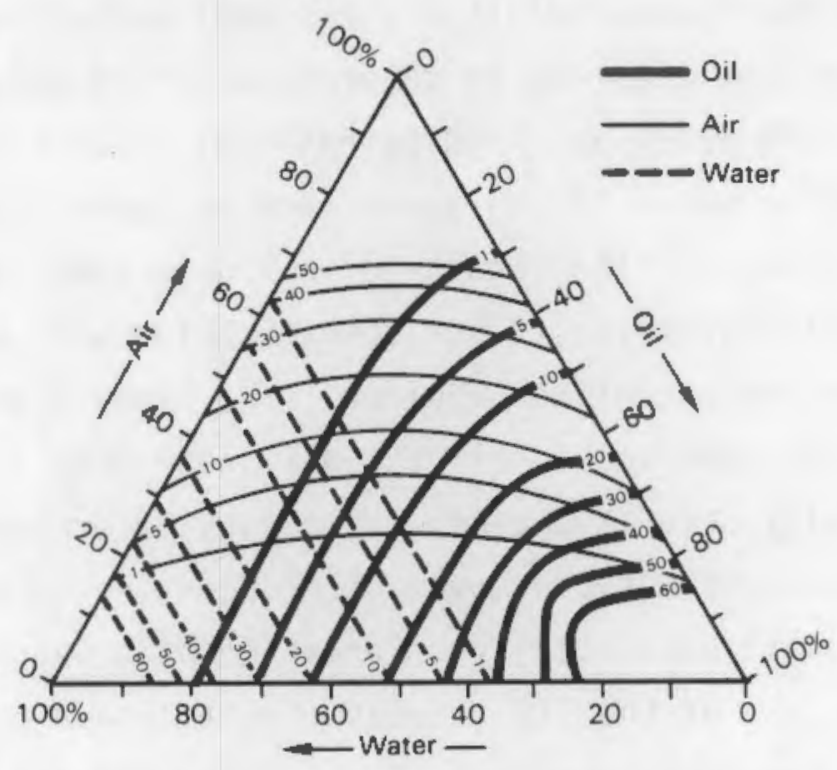

FIGURE 3.6. Relative Permeabilities for Three-Phase Flow (Van Dam 1967)

At low organic fluid saturations, a continuous organic phase may not exist. Such continuity is an essential assumption in virtually all existing models. However, organic compounds may exist as globules surrounded by water instead of as a continuous fluid phase and transport may be a result of globulemigration. Existing mathematical models and codes do not address this possibility and transport may be a result of globule migration.

\subsubsection{Physical Parameters}

Physical characteristics that play a determining role in the migration of chemicals are relative permeability, saturated-hydraulic conductivity, residual saturation, solubility, density, viscosity, and evaporation rate. Two- and three-phase diagrams for relative permeability must be developed in the laboratory for specific fluids and soils of interest. The saturated-hydraulic conductivities are required to scale the relative permeabilities to actual soils. A knowledge of residual saturation, which is fundamental to determining the capacity of the soil to retain the bulk chemical spill, must be determined for each chemical and soil combination. Surface tension must play a role in determining the residual saturation. However, current methods emphasize the 
measurement of the bulk parameter (i.e., residual saturation). The miscible or immiscible nature of the chemical is determined by its solubility in water. Fluids immiscible with water and held by residual saturation in the soil column will be transported by water in the subsurface at concentrations defined by their solubility in water. The density of a liquid chemical relative to the density of water will determine if the contamination will migrate at the upper or lower surface of the unconfined aquifer. The density of a chemical vapor phase relative to the density of soil air will determine if the vapor rises or falls in the partially saturated zone. Viscosity determines the velocity of the advective or convective fluid movement through its incorporation in the definition of hydraulic conductivity. Formation of a vapor phase, as defined by the evaporation rate of the liquid phase, will depend on physical characteristics, such as the vapor pressure of the fluid and its latent heat of evaporation.

In general, the data on physical and chemical parameters are not available for most chemical and soil combinations, and their absence represents a potentially significant time and financial investment for a chemical waste migration study. Data on the chemical attenuation of organic compounds and inorganic species influenced by organic compounds are also generally unavailable.

Darcy's equation, which is believed to provide the necessary mathematical equation for momentum balance for single-phase flow in a porous medium, is based on the representative elementary volume (REV) concept. Averaging theory (e.g., the REV) is important because it enables measurable properties at one scale to be related to parameters and predictions at another scale (i.e., pore scale, well-test scale, and large-scale heterogeneity). Recent research into the averaging theory has substantiated Darcy's equation; however, it has also introduced what are believed to be second-order influences that may significantly influence multiphase systems (Hassanizadeh and Gray 1980, Gray 1983).

Research into methods of characterizing the geologic environment and relating this characterization to subsurface flow and transport processes reveals that the scale of heterogeneity in the natural environment may be in conflict with scales of integration employed in volume-averaging techniques. Hence, existing deterministic equations of flow and transport may not be valid and 
stochastic methods (discussed in Section 3.6) may soon be viewed as essentia) in a comprehensive field characterization and modeling activity.

\subsection{RELEASES FROM A SOURCE}

Detailed characterization of the source release to the transport pathway is perhaps the most important element of any modeling effort; however, sufficient data are rarely available to enable mechanistic modeling of the source. Containment devices or structures that separate waste from the subsurface environment can significantly influence the release; the rate and duration of that release can significantly impact the transport process.

Empirically based fraction-remaining models are commonly used to quantify the release of contamination to be used as input to transport models. Whether mechanistic or empirical models of release are employed, they represent only the upstream boundary condition to the transport pathway. Accurate mechanistic simulation of the source release requires a knowledge of the waste form, including its phase (i.e., solid or liquid), chemical speciation, Eh, pH, and the existence of inorganic or organic complexants.

Most hazardous chemical and radionuclide releases are characterized as long-term events and are simulated with one-dimensional models. Onedimensional flow and transport conceptual models do not consider transverse dispersion, which causes a plume to be spread in the environment and reduces pollutant concentrations. Therefore, one-dimensional approaches may achieve conservative or upper-bound results and introduce additional conservatism through an overly simplified release model. An additional difficulty with source definition lies in the extrapolation of short-duration laboratory scale data to long-duration fieid-scale release scenarios (Claassen 1981).

\subsubsection{Isolation Afforded by Containment}

The mechanism that results in a pathway between waste and environment, such as the corrosion, degradation, or failure of any containment device or structure, must be considered in any release scenario. Release cannot occur before a mechanism acts to create a gradualiy increasing avenue of transport between waste and environment. For example, the corrosion of a steel container 
may expose waste to soil (and/or water table if a humid site) via a gradually increasing surface area. - In a partially saturated soil environment, the gradual increase in surface area implies a gradual increase in contaminant release. In a saturated porous medium, the surface area of contact may be of less importance after a certain level of communication develops because the cross section may not act to limit the transport through (or flushing of) the container. Extrapolation of the failure of one container or structure to the failure of many may be required to model an entire site. Clearly, mechanisms resulting in communication between waste and environment may act to control the release of a contaminant to the transport pathway. In addition, models of source release need to consider mechanisms of release (e.g., desorption and dissolution) as well as containment failure (e.g., corrosion).

\subsubsection{Simple Models of Source Release}

The complex geochemical character of sources and the lack of data providing insight about dominant release mechanisms force one to use simple release models that are, at best, empirically based. Fraction-remaining models, which quantify the fraction of waste remaining in the source as a function of time, can be used to define releases. Another approach is to simply specify the flux (i.e., mass or activity per unit area) leaving the source. These two approaches are equivalent; however, the fraction-remaining model is nondimensional and more easily applied to a variety of waste constituents.

Plausible release mechanisms include desorption and dissolution (Claassen 1981). Another process that limits mass transfer from the waste form involves a diffusion limiting layer on the exterior of the waste form. Such a layer could result from chemical precipitation of a species not of special interest on the waste form surface. The decay of radioactive sources can also be readily incorporated into simple release models.

Release rate and duration can significantly influence the character of the transport simulation. The period of release is described as short-term when it is less than the travel time to the accessible environment and long-term when it exceeds this travel time. Short-term, solubility-defined releases can be attenuated by physical processes that spread the pulse in the subsurface environment. Longitudinal and transverse dispersion will act to reduce the 
maximum observed concentration after the source is depleted. Long-term, solubility-defined releases are not significantly attenuated because of the continuing supply of chemicals at maximum concentrations. For radioactive contaminants, the decay process will always act to reduce the concentration; however, for long-lived radionuclides this effect may be of relatively minor importance.

Use of the linear sorption isotherm (discussed in Section 3.5.1) to mode] geochemical attenuation will only result in a delay of the release. Often the linear isotherm model is described as the velocity retardation model. The linear sorption isotherm manifests itself in the advection-dispersion transport equation (discussed in Section 3.4) as a retarded velocity and retarded diffusion coefficient. Increases in the retardation factor result in decreases in the average contaminant pulse velocity. Thus, use of the linear sorption isotherm does not attenuate the source; it only serves to delay the contaminant arrival at the accessible environment.

\subsubsection{Mechanistic Models of Source Release}

Just as transport in the subsurface is the result of complex geohydrologic processes and geochemical reactions, the release from a source and its equilibration with nearby soil is also a result of complex interactions. In nearly all cases, the geochemical and hydrological aspects of the waste form are more severe than and as varied as those found in the natural environment. For example, leachate may exhibit extreme acidic or basic ph values, and the conductivity and porosity of the waste form may be unknown.

Knowledge of geochemical characteristics of the waste form as it ages within its disposal container is important. Treatment and management practice (e.g., removal of excess water during interim disposal) may reduce a liquid waste to a solid waste before final disposal. More subtle changes in the chemical speciation of the waste and the creation or existence of inorganic or organic complexants during the aging process can have significant impact on the mobility of the chemical wastes.

Significant changes may occur to the chemical composition of a leachate on contact with the soil environment. Soils normally have a buffering capacity 
that can significantly affect the $\mathrm{pH}$ of the leachate. The interrelationships between $\mathrm{pH}$, Eh, chemical speciation, adsorption/desorption, precipitation/ dissolution, and oxidation/reduction can then act to alter the chemical character of the advancing leachate front. To predict the complex aqueous composition of leachate in the waste and in the adjacent soils, a substantial body of data must be obtained on the geochernistry of the contacting liquids, mineralogy of waste and soil, and thermodynamic constants for the multitude of possible reactions.

\subsection{PHYSICAL PROCESSES: TRANSPORT}

Solute is transported in a porous medium by advection or convection with the resident fluid and molecular diffusion (Pratt and Jury 1984). The geometrical complexity of the pore space forces one to idealize the description of either the pore space itself, or the resultant velocity or discharge characteristics of the flow. In either case, the concept of dispersion is used to characterize variations from the average flow. The three processes of advection/ convection, diffusion, and dispersion are discussed briefly in the following paragraphs. These processes occur within each fluid phase, and the relative importance of each may vary within the different fluid phases.

\subsubsection{Advection/Convection}

Advective/convective transport refers to the horizontal and vertical coincident transport of fluid and solute within the pore space. By definition, this transport process only occurs in moving fluids. When fluid motion is caused by forces applied at the boundary of the system, the process is referre 1 to as forced advection. The transport of neutrally buoyant solute by a natural ground-water system is a forced advection process. In the case where fluid neither enters nor leaves the system, the process is called free. Density variations caused by either chemical composition or temperature can cause a vertical exchange or circulation of fluid; this is an example of free convection. The term buoyancy flow is often used to describe the density-driven, freeconvection process.

Fluid and its attendant solute are advected in the subsurface system at a rate equal to the seepage velocity of the fluid. Fluid velocity is commonly 
available only as a result of model predictions of pressure or hydraulic gradient. These predictions are based on spatially averaged values of media and fluid properties. The advective or convective component of the transport equation represents the rate at which solute is transported with the average seepage velocity. In reality, the velocity of the fluid exhibits considerable variability within the pore space. Thus, the flow path of the fluid and its attendant solute may differ from the average seepage flow path. The process of dispersion (see Section 3.4.3) is used to approximate the solute distribution that is observed about the mean flow path.

\subsubsection{Diffusion}

The diffusion of a tracer or solute in a fluid in the pore space is caused by the molecular motion of the solute. Diffusion always results in the very gradual spreading of the solute into an ever-widening volume of the fluid in the porous media. Experience indicates that the diffusion process is rather slow and the spreading is restricted to relatively short distances over very long periods of time. The driving force or causal mechanism is the random motion of the solute molecules. Hence, the solute will spread from where it is to where it is not in abundance. Molecular diffusion simply rearranges solute molecules in the fluid. The solute concentration gradient is a measure of driving force. In the presence of a concentration gradient, molecular diffusion transports solute toward regions of lesser concentration. Thus, diffusive flux is directly dependent on the negative of the concentration gradient. Molecular diffusion will occur in fluids that are moving or at rest.

\subsubsection{Dispersion}

In contrast to molecular diffusion, dispersion of a solute cannot occur in the absence of fluid motion. Unlike diffusion, the dispersion process is the result of microscale variations in the fluid velocity within the pore space and megascale variations in fluid velocity resulting from heterogeneity of the porous medium. The velocity variations, which may retard or hasten solute advance at different places or times (i.e., as compared to the mean movement), are the cause of dispersion. Dispersion does not occur in a static fluid because there are no velocity variations unless there is motion. The variations of fluid velocity in the porous media that cause dispersion can be 
discussed on the basis of microscopic pore scale variations of velocity and on the basis of larger macroscopic or megascopic scale variations.

On the microscopic scale of individual pores, the fluid velocity variations are caused by variability in the pore space geometry, the velocity profile in the pore, and the flow path tortuosity. A portion of the solute will be carried by higher fluid velocities, while another portion of the solute wil be transported by slower fluid velocities. Movement along the longer and more tortuous flow paths around soil particles in the porous medium further accentuates the solute spread. Variability in each of the three factors causes velocity differences that result in extending and broadening a solute plume. Similarly, the larger-scale macroscopic or megascopic dispersion is associate. with differing fluid velocities. The variability found in fluid velocities results from spatial changes or variations in hydraulic conductivity and effective porosity; i.e., velocity is caused by the heterogeneity of natural porous materials. Thus, macroscopic or megascopic dispersion is a result of large-scale variability arising from natural heterogeneities that cause variations in fluid velocities at the macro or megascale.

Careful examination of the diffusion and dispersion processes shows they are fundamentally different processes. Diffusion is driven by a solute concentration gradient and occurs whenever and wherever a gradient exists. It makes no difference whether the fluid is at rest or in motion because the driving force of the diffusion process is the solute concentration gradient. Note that the dispersion process gives rise to solute concentration gradients which, in turn, drive the diffusion process.

Dispersion occurs only when there is movement of the transporting fluid through the porous material. In the strictest sense, for dispersion to occur there must be variations in the fluid velocity carrying solute from one point to another. As already discussed, the porous medium by its very nature provides the velocity variations that result in dispersion. The velocity variations that retard and hasten the advance of solute are the sole cause of dispersion. Dispersion cannot be caused, induced, or driven by a concentration gradient. Solute, in a purely dispersive process, is only a traceable marker that is along for the ride and meticulously follows the transporting fluid. 
Admittedly, the result or consequence of the dispersion process is an observable concentration gradient. Nevertheless, dispersion-induced changes in the solute plume are strictly the result of fluid velocity variations.

Dispersion is commonly described as having two directional components, longitudinal and transverse. The longitudinal component acts in the direction of the mean fluid velocity and the transverse component is orthogonal (i.e., at right angles) in relation to the mean fluid velocity. One-dimensional models of transport phenomena commonly consider only the longitudinal component. Two and three-dimensional models consider both. Longitudinal dispersion acts to distribute the solute in the direction of fluid flow; however, long-term releases can attain maximum source concentrations in model simulations if only longitudinal dispersion is considered. Transverse dispersion acts to distribute the solute into adjacent fluid pathways, thus spreading the solute plume and reducing solute concentration in the principal solute pathway.

Large- and small-scale heterogeneity observed in geologic media make it unlikely that existing advection-dispersion models of transport can predict the true solute path in the environment. Indeed, the applicability of the advection-dispersion equation has been called into question (Dagan 1982). Hence, the extension of models of single-phase flow and transport to multiphase systems cannot be made with any real confidence that a predictive capability will be obtained.

Parameters for previously developed models of dispersion are being reinterpreted, and entirely new models for dispersion are being proposed based on interpretations of the spatial variability characteristic of the porous media. Methods of sampling and interpreting the heterogeneous character of the medium are important aspects of forthcoming changes in models of transport in the subsurface environment.

\subsection{CHEMICAL REACTIONS: GEOCHEMISTRY, DECAY, AND MICROBIOLOGY}

Persuasive arguments can be made for the development of more mechanistic geochemical models and their requisite data. The final product of such work would not be limited by the linear sorption isotherm model and would be more applicable and acceptable. Unfortunately, the extensive data base for such a 
model does not exist for the broad range of hazardous chemicals and soils of interest. Geochemists may find that heterogeneity poses difficulties for mechanistic geochemistry similar to those difficulties currently posed for solute transport. The spatial variability within the geologic environment implies uncertainty not only in the transport process but also in the geochemical character of the porous medium.

There are only two geochemical reactions that directly influence the solute concentration avallable for transport: adsorption/desorption and precipitation/dissolution. Other physicochemical processes, such as aqueous speciation and reduction/oxidation, act to control these geochemical reactions. Radioactive decay is an important consideration for radionuclides, and microbiological degradation is a potentially important consideration for organic compounds. Each of these processes and reactions is described below.

\subsubsection{Adsorption/Desorption}

The effects of adsorption/desorption of chemical elements on soils must always be considered because elements can be adsorbed even when they exist at very low concentrations. Adsorption is the process by which ions in solution adhere to the surfaces of the solid bodies they contact. Desorption, the opposite process, releases ions from the solid surface. Ion exchange is one model of adsorption and desorption in which ions on a solid surface are exchanged with ions of like charge in solution. Commonly applied models of adsorption/desorption are reviewed by Travis and Etnier (1981). Other more detailed models of adsorption are described by Kincaid, Morrey and Rogers (1984); however, the linear-sorption isotherm is the most commonly applied model of adsorption applied in subsurface transport models.

The linear-sorption isotherm is characterized by a constant distribution coefficient $(K d)$, which represents the ratio of the concentration of the contaminant in the solution phase to its concentration in the solid phase (Cherry, Gillham and Barker 1984). The operative assumption is that the two quantities are related by a constant or linear function. The actual exchange reaction is assumed to occur relatively fast and is known to depend on the thermodynamic activities of the exchanged ions and their adsorbed states. 
When contamination is present in very low concentrations and the exchange does not cause a significant change in the activity ratio, the distribution coefficient is constant and can be defined in terms of contaminant concentration instead of thermodynamic activity. A constant distribution coefficient allows the incorporation of adsorption into transport models. Also, more mechanistic models of adsorption/desorption commonly retain the activity formulation in order to simultaneously consider distinct reactions and processes (e.g., adsorption, desorption, precipitation, dissolution, oxidation, and reduction).

The linear sorption isotherm has 1 imitations with respect to heterogeneous geologic deposits, geochemical complexities, and dynamic transport processes. Heterogeneity of deposits poses a problem because the distribution coefficient is actually a function of the mineral composition of the porous media. Batch tests used to define the coefficient are routinely based on relatively few soil samples. These tests are also based on ambient ground water. The advancing contaminant plume continuously changes the background concentration, requiring a suite of batch tests to provide an approximation to observed sorption behavior.

The second limitation of the linear sorption isotherm is that the exchange properties of the porous medium, which are assumed to be temporally constant, are actually continuously altered; this results from the complex interaction of adsorption, precipitation, oxidation, reduction, and hydrolysis. Thus, accurate prediction of sorption behavior would require simulation of the dynamic exchange properties of the environment.

Finally, the dynamics of transport are commonly characterized by plots of concentration versus time called breakthrough curves. These curves are usually asymmetrical and have extended tails (i.e., long-term but low-level releases). Breakthrough curves produced by advection-dispersion models with the linear sorption isotherm are nearly always symmetrical. Failure of the linear sorption model to duplicate this characteristic transport trait suggests the presence of a factor or process that is absent from the current suite of transport and adsorption models. 
Other sorption isotherm models found in solute transport codes include tie Freundich and Langmuir isotherm models (Travis and Etnier 1981). The Freundich isotherm model is an experimentally based isotherm (as is the linear isotherm) that accounts for nonlinearity in the partitioning of the liquid anis solid. As is true for the linear-isotherm model, there is no saturation limi: for the sorbed species. The Langmuir isotherm model, which was originally derived for the sorption of gases by solids, considers the saturation of site'; on the solid. It was derived from a set of limiting assumptions (rather than experimentation) and requires knowledge of the saturation concentration on this solid and of the ratio of the adsorption and desorption rate constants, which depend on the chemistry of the host media.

Existing interpretations of solute transport and geochemistry are the basis of simple and idealized models of aquifer systems that conduct a single liquid phase. These same idealized models are applied to multiphase flow and transport, although data to support such modeling is lacking. Results of classical models can provide estimates of chemical migration; however, the final fate of the chemical will be in question until models, are improved and data assimilated. Simple and idealized geochemical models such as the linear sorption isotherm are based on restrictive assumptions that make them an unsatisfactory basis for future model developments.

\subsubsection{Precipitation/Dissolution}

The level of contaminant concentration in ground water is commonly influenced by the precipitation/dissolution reactions that occur in response to sol. ubility constraints. When the product of activities of the combination of aqueous species (e.g., the solubility product) exceeds a certain value, precipitation can result. This process may be strongly dependent on ph. For exarlple, if a concentrated-acidic aqueous solution is percolated into a geologic medium with large buffering capacity, it will dissolve minerals and at the samle time will be neutralized. When this happens, solubility products of some dissolved minerals will be exceeded, thus causing them to precipitate. As in the case of the adsorption/desorption reaction, equilibrium with respect to 
precipitation/dissolution is achieved through the competition of severai reactions and processes (e.g., adsorption, desorption, oxidation, reduction, hydrolysis, and complexation).

\subsubsection{Chemical Speciation}

The chemical speciation resulting from hydrolysis and complexation is important because most contaminants exist in the aquifer in more than one ionic form or species. Mobility and solubility are highly dependent on the ionic form. The linear-sorption isotherm model of chemical mobility characterizes the average mobility. Thus, if the dominant ionic form or species of an element is highly mobile, the distribution coefficient will be relatively low. On another occasion, when the highly mobile ionic form does not dominate, the distribution coefficient for the same element could be relatively high. Similarly, highly soluble ionic forms can significantly influence precipitation/ dissolution reactions. Thus, a knowledge of or an assumed definition of chemical speciation is necessary to be able to predict chemical migration.

\subsubsection{Dxidation and Reduction}

Redox processes are important to several inorganic chemicals that have more than one possible oxidation state. Elements influenced by redox that appear in drinking water standards include $\mathrm{As}, \mathrm{Cr}, \mathrm{Fe}, \mathrm{Hg}, \mathrm{Mn}, \mathrm{Se}, \mathrm{U}, \mathrm{N}$, and $S$. Other elements in the drinking water standards that are strongly influenced by redox processes, even though they have only one valence state in aqueous systems, are $\mathrm{Ag}, \mathrm{Cr}, \mathrm{Cd}$, and $\mathrm{Bn}$ (Cherry, Gillham and Barker 1984). Eh-pH diagrams are used as guides to the redox status of the aqueous systems; however, they do not lead directly to predictions of contaminant mobility. The redox status applicable to a contaminant is dependent on the major-ion chemistry as well as the Eh-ph relation.

\subsubsection{Radioactive Decay}

The nucleus of the atom is held together by attractive forces between the neutrons and protons. Radionuclides decay by emitting radioactivity. Early experiments disclosed three different types of radioactivity: alpha, beta, and gamma radiation. Alpha rays are charged helium ions, beta rays are negatively charged electrons, and gamma rays are high-energy electromagnetic waves 
(General Electric Company 1984). The half-life is the period of time in which half of the nuclei initially present in a given sample disintegrates through decay. The concentration decreases exponentially with time (Matthess 1984). 3.5.6 Microbiologicar Degradation

Enzymatic reactions of microbiological entities inhabiting the groundwater environment may be the most important transformation mechanisin for organic contaminants (Cherry, Gillham and Barker 1984). Active entities include bacteria, yeasts, fungi and viruses. Biodegradation of a broad range of organic compounds has been demonstrated (Bouwer, Rittmann and McCarty 1981, Kobayashi and Rittmann 1982). Compounds affected by degradation include pesti.cides, halogenated hydrocarbons, aromatic hydrocarbons, amines, and alcohols. The broad range of enzymatic activities in soils caused by the large mixed populations of microbial entities make it nearly impossible to predict active organic transformation pathways and rates. Few data pertinent to ground-water systems are available and the mechanisms of biodegradation for synthetic organic contaminants are not well understood (Cherry, Gillham and Barker 1984).

Models of flow and transport in the vadose zone do not commonly consider the mobility of the vapor phase. Both vaporization and gas production by microbiological degradation are potentially important to organic compounds. These processes and the mobility of their gaseous products are not considered in existing models because inorganic compounds are not influenced significanti., by similar processes. Therefore, these models may not be applied to organic compound cases. Methods of incorporating microbiological reactions and processes into single-phase and multiphase transport equations must be developed. The communication of organic species between phases and the generation of one phase by a reaction in another phase both act to complicate the reaction and process models previously applied to single-phase fluids.

\subsubsection{Fractured Media}

Analyses of dispersion and chemical processes for contaminant transport in fractured rock, such as shale, granite, basalt, or salt, are in the preliminary stage of development (Anderson 1984, Cherry, Gillham and Barker 1984). This type of analysis may be needed where deep-well injection of hazardous wastes 
can result in pollutant transport to the biosphere. Clay, which is also susceptible to fracturing, is used as a disposal medium for municipal, industrial, and low-level radioactive wastes. Therefore, hazardous chemical movement caused by the possible increase of flow velocities in fractured porous media over that seen in a uniformly porous medium is of major concern.

Mathematical models do not exist that are well accepted as representing single-phase flow and solute transport through saturated or partially saturated fractured media. Thus, while models of single-phase fluid in a porous medium are to some extent applicable to multiphase fluids in porous media, no such extension of mathematics and numerical methods can be made for applications to fractured media.

Recent developments in mathematical models of flow in fractures have been based on the concept of a dual porosity medium (Grisak and Pickens 1980). Flow in fractured porous media, which is a combination of flow in fractures and flow in porous media, can result in transient chemical concentration gradients between the water in fractures and pores (Cherry, Gillham and Barker 1984). Although the bulk of the flow occurs in the fractures, diffusion of trace organics and other chemicals into and out of the porous matrix can have a strong influence on contaminant behavior (Tang, Frind and Sudicky 1981, Grisak and Pickens 1981). This is caused in part by the fact that the surface area of the porous media is much larger than the area of the fractured surfaces that come into contact with the flow. Pollutants must come into contact with media surfaces before chemical interactions, such as adsorption, may occur. Porous media may also contain organic matter, which enters into reactions.

Anderson (1984) summarized the following conclusions regarding fracture flow from documented laboratory and fieldwork: 1) a significant retardation mechanism for pollutant transport in fractures is diffusion of the contaminants into the pores of the rock matrix; and 2) an acceleration mechanism for lowvelocity flow in fractures is dispersion within the water phase. Little is known, however, about the magnitude of the roughness of a fracture. Difficulties in describing the geometry and hydraulic characteristics of fractured rock in the field are the major delays in model development and testing. 
Cherry, Gillham and Barker (1984) report that the chemical reactions dur. ing contaminant transport can have very different effects on porous media and fractured rock (or fractured, fine-grained, nonindurated porous media). Advection theory and the isotherm approach to predictive transport modeling have been specifically developed for porous media. Measurement of adsorption parameters for predictive modeling for fracture flow in nonporous rock (such as. granite) is very difficult. The distribution coefficient must be defined in terms of effective surface area of reaction in the fractures instead of in terms of the mass of solids, as is done for porous media. Cherry, Gillham anc Barker (1984) state that few attempts have been made to determine these properties for undisturbed surfaces or to validate predictive models under field conditions.

\subsection{UNCERTAINTY ANALYSES}

Because our understanding of subsurface transport processes is undergoins: change, models and codes adopted to meet current needs must not be thought of as final. Waste management options must be analyzed with the best available technology based on the most accepted physical and mathematical interpretatiors of the environment; consenquently, it is important to pose models in a framework that yields results needed by decision makers and the public. The models may be based on simplifying assumptions, but results must lead to decisions that ensure the safe operation of waste isolation systems and the protection $c f$ the environment.

Single-valued, deterministic projections of ground-water flow and solute transport made with current technology will be replaced in the future with mean, variance, and percent-confidence projections based on statistical interpretations of the environment. Current technology applied to single-phase systems is oriented toward providing the mean or average response of the environment. The standard deviation or variance in the response is aiso needed.

Uncertainty in measured data and spatial variability occurring in the natural environment have been recognized as significantly influencing predictions of the ultimate fate of solute in the subsurface. Regulatory criteria for the safe disposal of wastes reflect the increasing uncertainty in estimates given 
for the short- or long-term performance of the geohydrologic system. This is indicated by requests for sensitivity analyses that bracket estimates of performance within a confidence interval. Such analyses complement the predicted performance of the system and provide the decision maker with essential information on the range of possible system performance.

Methods of providing uncertainty analyses in single-phase systems are only now being developed. Many of these methods are fundamentally related to statistical descriptions of geohydrologic properties. Basic data are lacking for simulating multiphase systems that generate the statistical models.

There exists considerable uncertainty with most variables used as input to estimates of source release rates, ground-water flow rates, and solute transport. The best estimates of input values to models may not represent the system as well as would the use of all available information, i.e., using a statistical representation of input conditions (GTC 1985). Statistical sampling methods applied to deterministic equations (e.g., random or Monte Carlo sampling) may be a more manageable, although less efficient, approach to uncertainty analyses than the governing stochastic differential equations. The Latin Hypercube Sampling (LHS) technique, described by Mckay, Conover and Beckman (1979), could be used when sampling input-variable distributions of known sample size. Sensitivity analyses are also useful in determining the influence of various input parameters on output variation.

The Geologic Testing Consultants (1985) described the value of using partial-rank correlation coefficients to assess input variable importance. The calculations of partial correlations on ranked data will reveal the monotonic (i.e., nonlinear) relationships between model input and output. This method would simplify uncertainty analyses by eliminating many of the unimportant variables from consideration. Correlation among input variables can also be imposed as a result of expert opinion and intuition. 


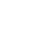

.

.

.

. 


\subsection{GUIDELINES FOR CODE SELECTION AND USE}

Computer modeling of ground-water flow and pollutant transport processes is helpful in organizing and analyzing site-characterization data for decisionmaking activities associated with DOE waste management systems. These activities include initial screening for new disposal sites, design of disposal facilities, design of monitoring networks and schedules, and prediction of environmental pathways for actual or hypothesized contaminant transport. If modeling is initiated early in a field program, it can be used to guide subsequent data collection activities. Modeling helps to gain a more quantitative understanding of the physical and chemical interactions taking place within an aquifer system by testing various interpretations that are based on limited field data.

This section presents a philosophy for code selection and use based on material that was adapted from Guidelines for Selecting Codes for Ground-Water Transport Modeling of Low-Level Waste Burial Sites (Simmons and Cole 1985a and 1985b). That report was written for the National Low-Level Waste Management Program funded by the DOE to provide guidance to managers and site operators who must select ground-water flow and transport codes and direct modeling activities for assessing shallow-land burial site performance. The guidelines were originally prepared for the selection and application of codes to be used in modeling single-phase flow systems. These guidelines apply equally well to multiphase and organic transport codes, although data for organics are lacking and few established codes are applicable to multiphase and organic contamirants. The guidelines are also applicable to hazardous chemical and high-level waste management because subsurface-contaminant transport problems are the same for chemical and high-level wastes as for low-level wastes. As for that program, it is also assumed that a professional geohydrologist with modeling experience will perform the modeling task after selecting the code and developing the conceptual model in cooperation with the site manager.

Site managers and operators must direct a modeling exercise by performing some basic tasks. First, specific study objectives, available funds, and the 
task schedule must be identified. Second, selection of the modeling approach and code must be done with the aid of professional modelers. Most importantly, site-specific data must be made available to the modelers to develop the sitespecific model to be used in simulations. The manager will coordinate all modeling activities and ensure quality assurance so that the results will carry scientific credibility. Quality assurance measures include chain-of-custody control on field samples, analysis results, and proper record keeping.

The model development activities to be performed by professional modelers (in cooperation with site managers) include defining study objectives, collecting or compiling site data, developing the conceptual model, identifying important processes at the site, selecting the code or codes with the required descriptive equations, coupling or interfacing codes if needed, evaluating code capabilities, verifying code performance, calibrating and validating the site model, performing simulations, and comparing the results with study objectives. All of these activities continuously feed into the formulation and revision of the conceptual model, as well as the computer model. This evolutionary process of conceptual modeling and code selection is illustrated in Figure 4.1. Aside from the difficulty in obtaining all the the necessary input data for a specific site, many simplifying assumptions must be made during conceptual-mode? development to permit mathematical modeling. However, a simplified model can obtain meaningful results if important characteristics of the system are preserved. An experienced and skilled ground-water hydrologist is required to apply a code that will retain the essential processes in the conceptual model of the system.

The selection of a computer code or codes for modeling a ground-water flow system and pollutant transport processes must be based on the conceptual model for the specific site and waste disposal practices. Development of the conceptual model requires a clear understanding of the study objectives and compilation of available field data. The conceptual model is a simplification of the actual system because a complete data set is never available (e.g., property variations in all three dimensions) and reasonably complete data sets are not 


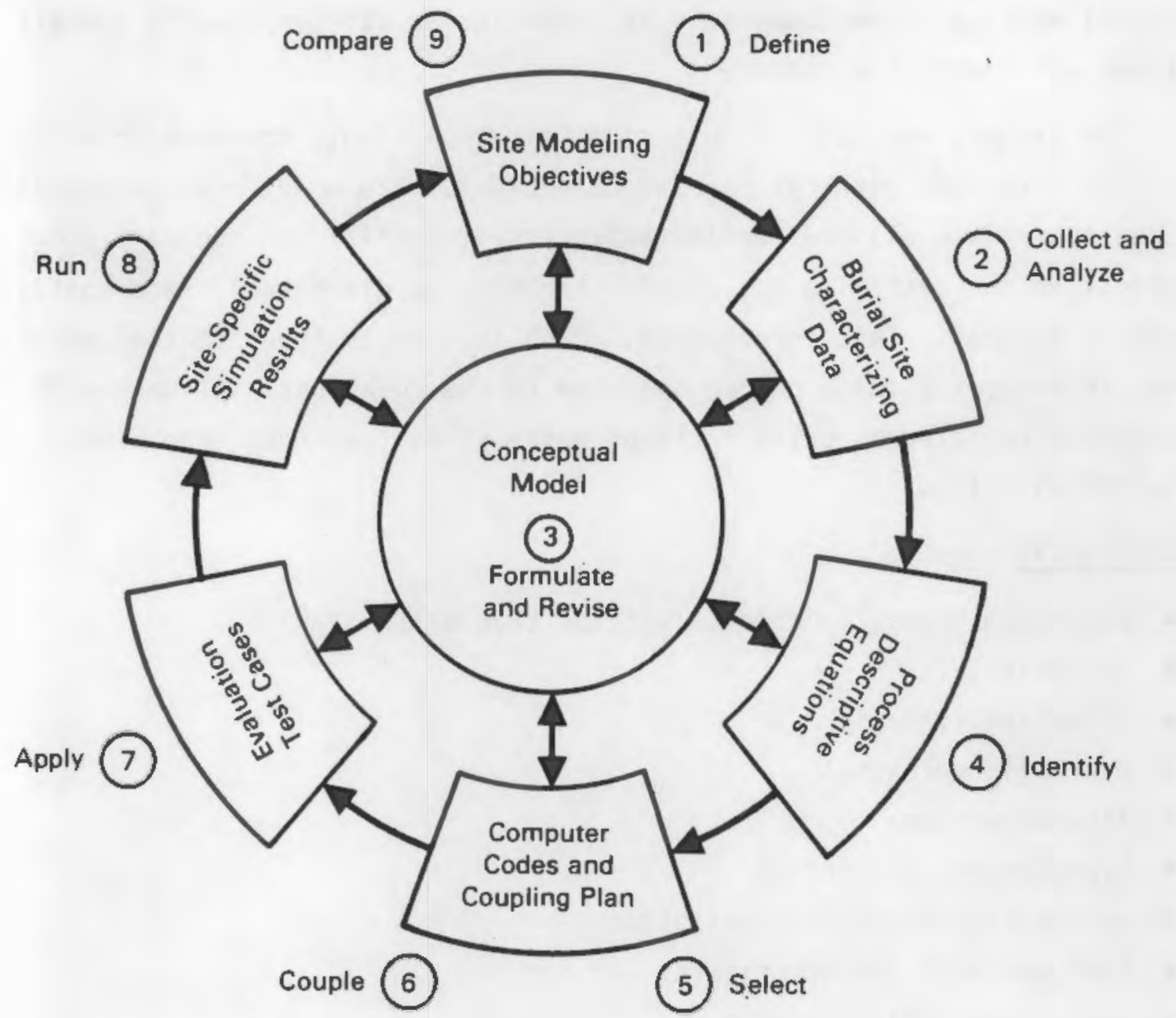

FIGURE 4.1. Systems Model Components (Simmons and Cole 1985a)

available at most sites. An extensive monitoring program of many wells would be needed to completely define all property variations in all three dimensions over time. Therefore, assumptions must be used to simplify the conceptualization of the system, yet not interfere with the study objectives. The objectives will help to define the type of output and processes required from simulations. Site data must be reviewed for completeness to establish whether 
the required process simulations are possible. For example, if it is important to model vertical flow components at a particular site, hydraulic property variations with depth are required.

The assumptions used in this simplification (i.e., the conceptual model) must not interfere with the problem objectives. The model must be detailed enough to include all the pertinent processes, initial and boundary conditions, stresses on the system (e.g., aquifer recharge or discharge), and contaminant sinks or sources. This information, which is used as input to the computer code, is necessary for a unique solution to the equations. A summary of common parameters required in characterizing waste sites (expanded from Simmons and Cole 1985a) follows:

Hydrological

- saturated hydraulic conductivity or transmissivity

- storativity

- effective Porosity

- hydraulic potential

- flow direction

- hydrodynamic dispersion

- precipitation/evapotranspiration

- hydrogeologic characteristics

- soil characteristic curve

- unsaturated hydraulic conductivity curve

- water content

Geochemical

- ion exchange capacity

- sorption isotherm models

- soil pH, Eh

- soil mineralogy

- surface water chemistry

- aqueous geochemistry (natural and impacted) 
Geotechnical

- soil classification

- compaction relation

- grain-size distribution

- density

- strength.

The collection of additional data or changes in objectives may modify the conceptual model and therefore require changes in the computer code or in the application of the code in modeling the system. See Simmons and Cole (1985b) for detailed discussion of all modeling steps. Simmons and Cole (1985a) recommended that a small group of codes (e.g., those applicable to various ground-water flow and transport problems) be rigorously evaluated for capabilities, fully documented, and organized for coupiing to one another according to the conceptual model of the site. 
.

.

. 


\subsection{OVERVIEW OF AVAILABLE COOES}

This section presents information on codes available for modeling groundwater flow and solute transport. First, some existing reviews are briefly described, and the number of codes cited in each is tabulated. Because current reviews exist, this review will not attempt a detailed evaluation or categorization of all available codes. Second, five documented and extensively used codes are described. Each of the five codes represents a distinct computational capability. None of the available codes of similar capability are necessarily the best; they are simply representative of those available.

\subsection{PUBLISHED REVIEWS OF COOES}

Table 5.1 summarizes the number of codes cited in recent model review documents for modeling the various flow and transport processes. These reviews are briefly described below.

Van der Heijde, et. al., (1985) reviewed 399 codes from 19 countries that are believed to represent as much as $80 \%$ of all codes available for modeling flow and transport of pollutants in surface water and ground water. The brief code descriptions in that document include the identification of the code and its development team, the current contact for obtaining the code, the purpose of the code, its conceptual and mathematical framework, information on the computer code and its implementation requirements, model applications and availability, and pertinent literature references. The objective of the van der Heijde review was to familiarize the technical reader with the essential conceptual and mathematical details and the state of usability of each of the surveyed codes. The codes were subdivided based on characteristics such as the purpose of the model, the water system treated (whether ground water alone or ground water and surface water), the level of spatial detail (lumped or distributed type models), and the management task that the model addresses. The management tasks were grouped in accordance with three problem areas, namely, water supply (quantity), contamination (quality), and environmental impact. The surveyed codes were also reviewed in terms of their accessibility (including documentation), availability to potential users, use support, and 
TABLE 5.1. Number of Codes Avajlable for Modeling Various Flow and Transport Processes (as Cited in Other Model Review Documents)

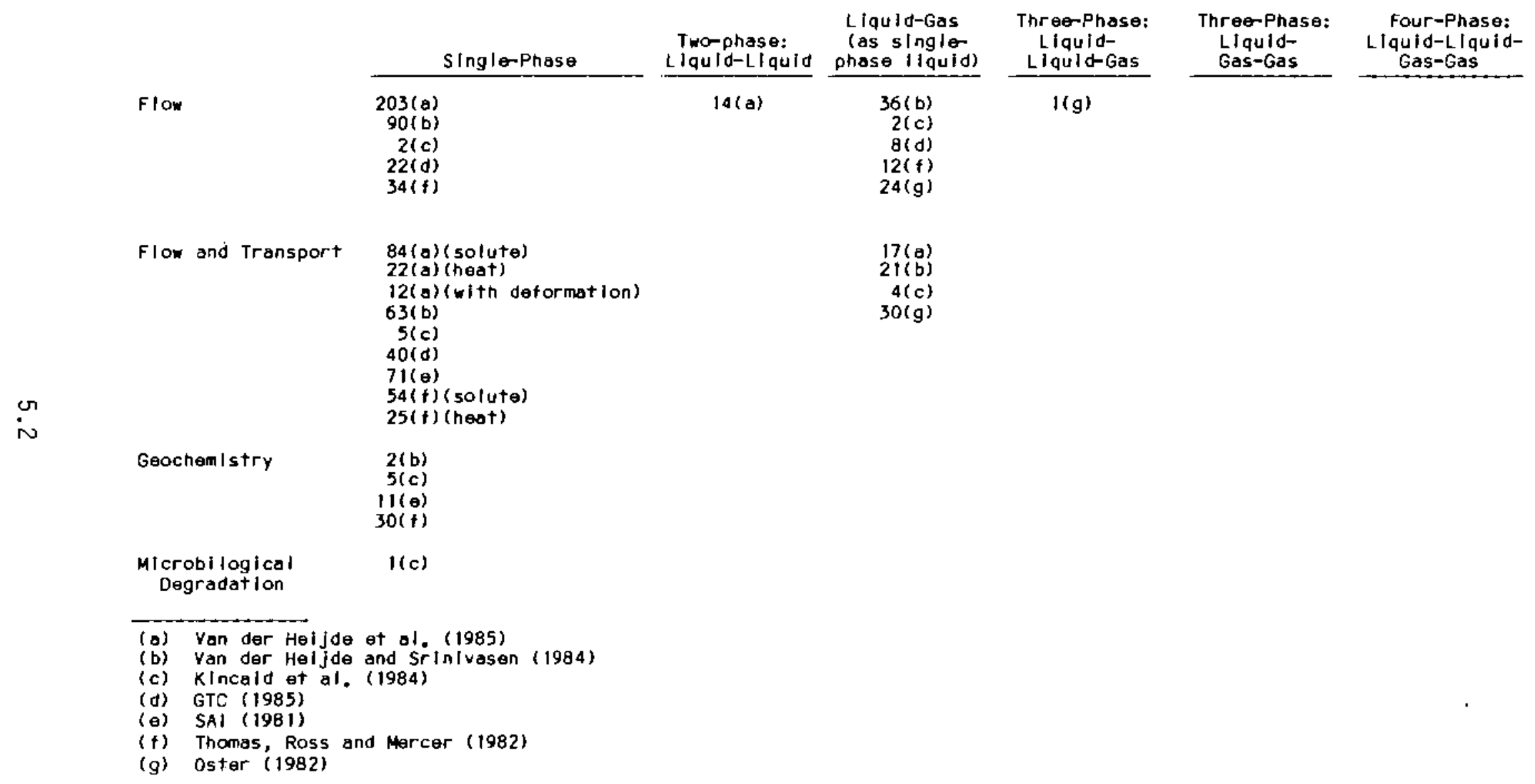


past applications. In this context, a code is defined as usable if users with modeling experience, other than the code developer, have applied the code effectively. In addition, successful application of the code to one or more field problems increases the degree of confidence placed in that code. To this purpose, the codes reviewed are available, well documented, and supported. The review cited 203 single-phase flow codes, 84 single-phase solute transport codes, 22 single-phase heat transport codes, 12 single-phase transport codes with deformation of the porous medium, 14 liquid-liquid, two-phase flow codes, and 17 liquid-gas, two-phase transport codes.

The Geologic Testing Consultants (1985) reviewed computer codes capable of simulating the various processes that determine radiation dose rates caused by shallow disposal of radioactive wastes. This included review of codes applicable to transport pathways in unsaturated and saturated ground-water systems. All codes reviewed were documented and nonproprietary. References for review documents, which included model verification descriptions, were included in the brief model summaries when available. The nonexhaustive review includes summaries of 40 geochemical or solute transport codes, 8 unsaturated flow codes, and 22 ground-water flow codes.

Van der Heijde and Srinivasen (1984) summarize hydrochemical codes available from the Holcomb Research Institute at Butler University in Indianapolis, Indiana. This list included approximately 90 single-phase flow codes, 36 liquid-gas, two-phase flow codes, 63 single-phase transport codes, 21 liquid-gas, two-phase transport codes, and 2 geochemistry codes.

Kincaid, Morrey and Rogers (1984) summarized 24 unsaturated flow and transport codes, 36 saturated ground-water flow and transport codes, 10 equilibrium geochemistry codes, 6 microbiological codes and a one-dimensional saturated flow, solute transport, and chemical equilibrium speciation code. Indepth reviews for 7 saturated and 6 unsaturated ground-water flow and transport codes, 5 geochemistry codes and 1 microbiological code were reported in Kincaid et al. (1984).

Science Applications Inc. (1981) tabulated descriptions of over 400 computer models applicable to waste isolation problems. The compilation includes categorization of the models according to their intended use and a 
description of the possible relationships existing among the models. The categories (and respective number of codes described) applicable to groundwater flow and transport simulations are geochemistry (11) and geosphere transport (71).

Thomas, Ross and Mercer (1982) developed critical sumaries for a variety of codes that are being developed by the U.S. Nuclear Regulator Commission (NRC) as candidates for benchmarking codes. The NRC requires quality-assured codes to support high-level waste management decisions. Specificaliy, codes are used in performance assessment of proposed nuclear waste management systems. The total number of codes reviewed in areas associated with the ground. water pathway is 155. These codes are divided into the following model/code types: 34 saturated-flow, 12 unsaturated-flow, 54 solute-transport, 22 heattransport, 3 energy- and solute-transport, and 30 geochemistry codes.

Oster (1982) catalogued 55 codes for use in modeling partially saturated flow and transport in porous media. Ten of these codes were examined in detail, although none were actually run on a computer for the study. The 0ster review includes discussions of the moisture movement and contaminant transport problem and methods used to reduce physical problems to a computer model. Input data requirements and the suitability of each of the 10 codes for various applications are also described by Oster (1982).

\subsection{SELECTED COOE DESCRIPTIONS}

Selected codes are summarized in Table 5.2. The following subsections include brief narrative discussions on the capabilities, input requirements, and limitations, as cited in the available documentation. Each code is representative of a group of codes that provide the same computational capability.

\section{2 .1 UNSAT 2}

UNSAT 2 is an improved and extended version of the UNSAT 1 code previously developed for solving problems of nonsteady seepage in saturated-unsaturated porous media by Neuman, Feddes, and Bresler (1974). The program can be used to investigate problems involving two spatial dimensions in the horizontal or 
TABLE 5.2. Summary of Capabilities for Selected Codes

\begin{tabular}{|c|c|c|c|c|c|c|}
\hline & Code & UNSAT2 & FEMWASTE & USGS3D & $\mathrm{MOC}$ & CFEST \\
\hline & Reference & $\begin{array}{l}\text { Davis and Neuman } \\
(1983)\end{array}$ & $\begin{array}{l}\text { Yeh and ward } \\
(1981)\end{array}$ & $\begin{array}{l}\text { Trescott }(1975), \\
\text { Trescott and } \\
\text { Larson }(1976) \text {, } \\
\text { Torak (1982) }\end{array}$ & $\begin{array}{l}\text { Konlkow and } \\
\text { Gredehoeft }(1978) \text {, } \\
\text { Tracy }(1982)\end{array}$ & Gupta, ot al. (1982) \\
\hline & Purpose/Output & $\begin{array}{l}\text { Translent Flow in } \\
\text { Partlaliy Satu- } \\
\text { rated Systems }\end{array}$ & $\begin{array}{l}\text { Solute Transport } \\
\text { in Partlally } \\
\text { Saturated Sys- } \\
\text { tems }\end{array}$ & Ground-water Flow & $\begin{array}{l}\text { Translent Solute } \\
\text { Transport }\end{array}$ & $\begin{array}{l}\text { Ground-water Flow } \\
\text { Transient Solute and } \\
\text { Heot Transport }\end{array}$ \\
\hline & $\begin{array}{l}\text { Fiuid Phases Simulated } \\
\text { Single Phase (Liquid) } \\
\text { Two Phase (Liquid-Gas) } \\
\text { Two Phase (Liquid-Liquid) }\end{array}$ & $\begin{array}{l}\text { yes } \\
\text { no } \\
\text { no }\end{array}$ & $\begin{array}{l}\text { yes } \\
\text { no } \\
\text { no }\end{array}$ & $\begin{array}{l}\text { yes } \\
\text { no } \\
\text { no }\end{array}$ & $\begin{array}{l}\text { yes } \\
\text { no } \\
\text { no }\end{array}$ & $\begin{array}{l}\text { yes } \\
\text { no } \\
\text { no }\end{array}$ \\
\hline r & Transport Phenomena Simulated & none & miscible solute & none & miscible solute & $\begin{array}{l}\text { miscible solute, } \\
\text { heat }\end{array}$ \\
\hline & Number of Dimensions Possible & $\begin{array}{l}2(3 \mathrm{axl}- \\
\text { symmetrlc) }\end{array}$ & 2 & 3 & 2 & 3 \\
\hline & $\begin{array}{l}\text { Numerlcal Techn Ique } \\
\text { FInIte Difference, Strongly } \\
\text { Implicit Procedure (SIP) } \\
\text { Finlte Element, GalerkIn } \\
\text { Formulation }\end{array}$ & $\begin{array}{l}\text { no } \\
y \in s\end{array}$ & no & $\begin{array}{l}\text { yes } \\
\text { no }\end{array}$ & $\begin{array}{l}\text { yes } \\
\text { no }\end{array}$ & no \\
\hline & SImulates Decay of Radionuclides & no & yes & no & yes & yes \\
\hline & $\begin{array}{l}\text { Sorption Simulation } \\
\text { Linear Isotherm } \\
\text { Langmulr Isotherm } \\
\text { Freund Ich Isotherm }\end{array}$ & $\begin{array}{l}\text { no } \\
\text { no } \\
\text { no }\end{array}$ & $\begin{array}{l}\text { yes } \\
\text { no } \\
\text { no }\end{array}$ & $\begin{array}{l}\text { no } \\
\text { no } \\
\text { no }\end{array}$ & $\begin{array}{l}\text { yes } \\
\text { yes } \\
\text { yes }\end{array}$ & $\begin{array}{l}\text { yes } \\
\text { no } \\
\text { no }\end{array}$ \\
\hline
\end{tabular}


vertical plane (Davis and Neuman 1983). Three-dimensional problems can be handled provided that the flow pattern retains an axial symmetry around the vertical coordinate. The flow region may consist of arbitrarily arranged, varying soil materials. The principal hydraulic conductivities may be oriented at any desired angle with respect to the coordinates.

Time-dependent boundary conditions that may be simulated include: prescribed pressure head, prescribed flux normal to the boundary, seepage faces, and evaporation and infiltration boundaries. The program can also model water uptake by plants as determined by atmospheric, soil, and plant conditions. Internal volumetric sinks or sources of prescribed strength can be included in the flow system at any stage of the computation. A special provision has been made for the analysis of axisymmetric flow to a well of finite radius, which partially penetrates an unconfined aquifer system and discharges at a prescribed time-dependent rate. The well may be partially cased and its capacity for storing water is taken into account. The influence of water withdrawal on several stratiographic layers that lie above, below, or within the screened interval can be analyzed.

UNSAT 2 is very flexible in modeling various-shaped flow regions. This is because one of three coordinate systems may be adopted ( $x-y$ cartesian, $x-z$ cartesian, and $r-z$ cylindrical) and because the actual boundary of the flow domain can be simulated using linear-triangular elements.

Inputs to UNSAT 2 include

- grid geometry

- initial heads (total head or pressure head)

- boundary conditions

- rates of infiltration, evaporation, and transpiration

- root effectiveness function

- hydraulic conductivity tensor

- relative permeability function

- capillary pressure function

- storage coefficient. 
The printed output of the program consists of a listing of all input information, a complete description of the finite element network, the boundary codes of all nodes, and the properties of each material. During each time step, the program prints a listing of total head values, pressure head values, moisture content values, and discharge into or out of (not flow through) the system at all nodes. The rate of convergence of the iterative procedure is printed during each time step, together with additional information pertaining to the particular problern at hand.

\section{2 .2 FEMWASTE}

FEMWASTE (A Finite-Element Mode1 of Waste Transport Through SaturatedUnsaturated Porous Media) was developed by Yeh and Ward (1979) to mode1 miscible solute transport through porous media under dynamic soil water and groundwater conditions. It is an extension of work done by Duguid and Reeves (1976). This code simulates the spatio-temporal distribution of both the solute concentration and flux under dynamic ground-water conditions. The subsurface system may have both saturated and unsaturated regions. Transport mechanisms modeled in FEMWASTE include advection, hydrodynamic dispersion, chemical sorption, and first-order decay.

The aquifer to be modeled may be simulated in two dimensions in areal $(x, y)$ or cross-sectional $(x, z)$ coordinates. The aquifer parameters may be distributed or zoned, and the system may be anisotropic. The code takes sorption into account by introducing a retardation factor into the governing massbalance equation. Nonlinearities are also considered insofar as the bulk density, and consequentiy the retardation factor, are a function of the moisture content. Velocity and moisture content fields are required as input to this code. FEMWATER, the companion code to FEMWASTE, produces the required velocity and moisture content fields. FEMWATER is not summarized here because it is based on the same physics as UNSAT 2, and its capabilities and input requirements are very similar to those of UNSAT 2.

Inputs to FEMWASTE include

- velocity and moisture content fields

- distribution coefficient 
- bulk density

- longitudinal and transverse dispersivities

- decay constant

- effective porosity for each material

- initial conditions

- boundary conditions

- grid geometry.

Output for FEMWASTE consists of the concentration distribution at each time step.

5.2 .3 USGS3D

The USGS3D code was developed to simulate ground-water flow in three dimensions (Trescott 1975). The code can be used in a two-dimensional mode, a fully three-dimensional mode or a quasi three-dimensional mode. It was developed at the United States Geological Survey and is described in a series of open-file reports (Trescott 1975; Trescott and Larson 1976; Torak 1982).

USGS30 simulates flow in a porous medium that may be heterogeneous and anisotropic and have irregular boundaries. The uppermost hydrologic unit may be an unconfined aquifer. The stresses considered are wells and recharge fror precipitation. One or more layers of nodes can be used to simulate each hydrogeologic unit. If it is reasonable to assume that storage is negligible in a confining bed and that horizontal components of flow can be neglected, the effects of vertical leakage through a confining bed can be incorporated into the vertical component of the anisotropic hydraulic conductivity of adjacent aquifers.

The data required to run USGS3D includes grid layout and control parameters for the finite-difference algorithms and physical data. The physical data include

- initial head distribution

- boundary conditions

- storage coefficient distribution

- transmissivity distribution

- recharge rates. 
If the upper unit is unconfined, hydraulic conductivity and elevation of the bottom of the water table aquifier replace transmissivity as input requirements.

USGS3D output includes all input, time-step information, mass-balance information, matrix-iteration information, and computed hydraulic head or drawdown.

\section{$5.2 .4 \quad M O C$}

The original MOC code documented by Konikow and Bredehoeft (1978) simulates transient changes in the concentration of a nonreactive solute in flowing ground water. The computer program solves the ground-water flow equation, which governs the head distribution in the aquifer, and the solute-transport equation, which governs the chemical concentration in the system. By coupling the flow solution with the solute-transport equation, MOC can be applied to both steady-state and transient-flow problems.

The MOC code, as modified by Tracy (1982), incorporates decay (a simple rate-controlled reaction) and general equilibrium sorption (an equilibrium controlled exchange) into the transport model. This code provides a means of assessing the movement of conservative, sorbing, and decaying constituents. Obtaining the parameters for the decay mechanism is straightforward as the phenomenon is well reported in the literature for radioactive species (General Electric Company 1984). The parameters for any of the adsorption models are not necessarily transferable from one field site to another. The chemical nature of the phenomenon is complex, and the adsorption model parameters are interdependent on the chemistry of the host aquifer. Frequently, these parameters must be determined from available field data for a given constituent.

The nonlinear sorption models have been implemented in an explicit, reasonably accurate manner. However, problems of accuracy can develop, particularly in regions of initially low concentrations that have rapid increases in concentration resulting from large source terms; mass is still preserved in these regions. However, the initial distribution is simply in error and will be redistributed over subsequent time steps. 
The most difficult user problem of the MOC code is the inability to control or to eliminate mass-balance errors induced by the existing particletracking scheme. This problem is magnified by the adsorption mechanism as a loss of mass in the solute state and is multiplicative by the equivalent retardation factor in the adsorption state. This problem can only be overcome by introducing a volumetric form of the transport equation and assigning and tracking volumes to all particles (Tracy 1982).

The input data requirements are time-step size, grid spacing and size, recharge, discharge rates and locations, effective porosity, storage coefficient, dispersivities, decay half-life, data for the desired sorption isotherm, transmissivity distribution, saturated thickness, and initial and boundary conditions.

The output from MOC includes hydraulic head distributions with mass balance, solute concentration distributions with chemical mass balance, and observation well data.

\section{2 .5 CFEST}

The CFEST (Coupled Fluid, Energy, and Solute Transport) code was developeC to determine the response of a confined aquifer to thermal energy storage. This code employs a standard Galerkin finite element method in the solution of the coupled equations of mass, energy, and solute mass conservation (Gupta et al. 1982). A sequential solution algorithm is used to solve the flow of water, the transport of energy, and the transport of solute. The finite element approximation to the continuum is made with a bilinear, two-dimensional, quadrilateral element that is simply expanded to the trilinear, eight node brick when three-dimensional analysis is warranted. CFEST can be used to analyze two-dimensional vertical or horizontal planes, two-dimensional axisymmetric cross sections, and fully three-dimensional aquifer situations.

The input required by CFEST, depending on the problem, includes hydraulic conductivity or transmissivity distributions for each geologic unit, porosity, medium compressibility, specific storage, thermal conductivity and heat capacity. Initial conditions of hydraulic head, temperature, and solute concentration are also required. Grid point locations for a finite element grid are 
required. Boundary conditions must be specified on the perimeter of the ground-water system, at intersections with surface water bodies or liquid waste disposal areas, and for injection or production wells.

The output from CFEST includes distributions of hydraulic head, temperature, and concentration as well as mass balance information.

\subsection{APPLICABILITY OF AVAILABLE CODES}

Codes described in the preceding section are a result of interest in overall moisture and ground-water movement, and transport of a solute that is miscible in water. It is apparent from our discussion of physical processes and chemical reactions that these codes are applicable to only a small, yet important, subset of multiphase flow and transport problems. These currently available codes could form the basis of a modeling capability for miscible organic compounds if additional mechanisms could be included in the existing model formulations. For example, microbiological degradation, volitilization, and adsorption-desorption submodels may be needed before existing codes could simulate organic compounds of interest.

None of the available codes described previously can address pollutant migration as a result of two immiscible fluids. Certainly, some codes do exist; however, they are either proprietary or the result of very recent research. As a result, no two- or three-phase models of fluid movement or pollutant transport are described in any detail. This is an important omission in available technology because it limits our ability to describe quantitatively the migration and fate of bulk organic liquid spills. Virtually any situation resulting in two or more distinct fluid phases is beyond the scope of currently and readily available codes. Examples of two-phase systems are soil water and soil air, and soil water and liquid organic compound. A three-phase system could involve soil water, liquid organic compound and soil air. In all cases, each phase is assumed to have a viable transport pathway. The inability of available codes to address these multiphase systems impacts our ability to quantitatively describe any multiphase pollutant problem.

Pollutant releases involving solutions that are immiscible in water must be modeled as multiphase systems. At the leading edge of a pollutant plume, 
where the immiscible solution is in contact with the soil water or ground water, one can expect some level of contamination of the water. This relatively low level of contamination in the water can be addressed by the miscible-solute transport codes. Movement of the bulk-immiscible pollutant. cannot be addressed. Recognize that the bulk pollutant is the source of the miscible solute. As the bulk pollutant migrates further into the subsurface system, an increasing cross-sectional area of soil and ground water is exposec to the pollutant. Thus, an inability to describe the immiscible pollutant migration casts a considerable uncertainty on a prediction of first release $t c$ the accessible environment based solely on a miscible solute transport model. In addition, the final fate of pollution, which is immiscible in water, can only be estimated by a multiphase model. The length of time required for a bulk spill of immiscible pollutant to either migrate through or disperse in tre environment can not be predicted with available models or codes.

To completely assess the potential for multiplase pollutant migration, a minimum of four distinct flow and transport models/codes are needed. These codes would address 1) one fluid phase, 2) two liquid phases, 3) one gas and one liquid phase, and 4) one gas and two liquid phases. Currently, codes are readily available to address the one fluid-phase problem, but even these codes may require the inclusion of significant processes and reactions before they can be applied to pollutants of interest to the DOE. 


\subsection{RECOMMENDATIONS}

Considerable scientific study is currently devoted to processes and reactions and their mathematical formulation in micro, macro, and megascales. A decision must be made whether fundamental, conceptual, and mathematical models of physical, chemical, source term, and microbiological models should be advanced before proceeding with multidimensional and multiphase flow and transport software (i.e., computer code) development. A modeling capability developed in response to regulatory issues should not only predict an expected response but also its uncertainty. Options include 1) advancing our knowledge of conceptual and mathematical models; 2) advancing deterministic models/codes to address true multiphase transport phemonena and to predict expected response and confidence intervals based on present knowledge, deterministic models, and advanced computational methods; or 3) elements of both of the preceding options. The third option is the only reasonable plan to execute. A better understanding of processes and reactions, improved models (e.g., stochastic) based on measurable parameters, and models that include uncertainty estimates are each essential. Existing computational codes must be expanded and improved to provide an interim simulation capability for complex multiphase flow and transport. Advances in all of these areas are needed to answer questions posed by the complexities of organic transport in multiphase systems.

As the investment is made to provide an advanced capability to predict multidimensional and multiphase system behavior, an expedient solution based on current knowledge must be provided for interim management problems. Interim codes also provide a basis for the development of the next generation of codes (e.g., statistical interpretations of the environment, Monte Carlo simulations yielding expected values and uncertainty). Unfortunately, initial estimates of system performance must rely on process and reaction models and deterministic formulations that may be judged inappropriate and, therefore, inadequate in the near future.

Initial efforts to apply existing single-phase fluid flow and transport codes to cases of miscible organic transport may require modifications to account for significant organic transport processes. These initial 
applications will certainly require extensive calibration efforts. In particular, the degradation and vaporization processes could be very significant in estimating the migration and fate of some organic chemicals. Calibration to organic transport events may not be possible because of a general lack of data. However, calibration to release events involving both organic and inorganic constituents should be undertaken on the basis of inorganic solute data. Because some organic chenicals are carcinogenic at very low concentrations, calibration efforts should examine the precision and sensitivity of simulations.

Efforts to analyze immiscible organic transport should be undertaken to provide estimates of system performance based on reasonable simplifications. A significant data base of physical, chemical, and microbiological properties relevant to specific organic compounds will he necessary to employ even simplified models. Simple models based on the retention capacity of the fluid and soil and the velocity of a bulk organic fluid should be developed to provide estimates of migration rate and location in the subsurface. Solubility and soil water-ground water flow rate could be used to estimate the long-term character of a chronic organic chemical release. More detailed and comprehensive analyses of multiphase immiscible flow and transport in both the partially saturated and saturated aquifer environment will probably be necessary to determine with any certainty the ultimate fate of these chemicals.

The types of hazardous and organic chemicals stored and used at Hanford sites and the potential health effects of these chemicals should determine the level of effort given to miscible and immiscible transport analyses. If organic chemicals immiscible in water are not stored or disposed in large volumes, then the need to be able to analyze their potential impact as an immiscible fluid may be significantly less than the need to be able to analyze for their miscible transport in soil water and ground water. Aspects of Hanford aquifers that may present opportunities for the isolation or attenuation of organic contaminants should also be identified. Examples of sitespecific considerations include 1) microbiological entities that consume organic compounds and either sorb them to soils or convert them to new organic compounds, 2) geologic deposits (e.g., clays) that may contain higher levels of 
microbiological entities, and 3) impermeable pockets in the geology that could act to retain organic contamination, which is heavier than water.

The research and development necessary to provide an interim, but potentially flawed capability now and a completely defensible capability in the future, will require substantial time and monetary resources. Clearly, the existing modeling capability for single-phase, ground-water systems cannot be extended to adequately address many aspects of organic chemical transport. Neither will the petroleum industry capabilities in this area address the transport issue. A broadly based program is needed to address the potential for liquid organic compound contamination of soils and aquifers at Hanford sites. Current needs to provide estimates of the fate of chemicais in the environment may require the expedient use of existing codes, which do not include all relevant processes and reactions. New methods of field sampling to determine dispersivity measures are being developed in conjunction with new interpretations of the subsurface environment. Certainly, these methods, conceptual interpretations, and models will be applied first to miscible organic compounds in single-phase systems. In this decade, methods of interpreting processes, sampling the environment, and analyzing transport in single and multiphase systems will all undergo significant change. Investments in technology to provide results in the near term must be made with an awareness of the evolution toward more mechanistically based and defensible models and codes applicable to multiphase flow and transport phenomena. 
.

. 


\subsection{REFERENCES}

Anderson, M. P. 1984. "Movement of Contaminants in Groundwater: Groundwater Transport-Advection and Dispersion." In Groundwater Contamination, Studies in Geophysics, National Academy Press, Washington, D.C.

Bouwer, E. J., B. E. Rittmann and P. L. McCarty. 1981. "Anaerobic Degradation of Halogenated 1- and 2-Carbon Organic Compounds." Environ. Sci. Technol. $15: 596-599$.

Cherry, J. A., R. W. Gillham and J.F. Barker. 1984. "Contaminants in Groundwater: Chemical Processes." In Groundwater Contamination, Studies in Geophysics, National Academy Press, Washington, D.C.

Claassen, H. C. 1981. "Conceptual Models Governing Leaching Behavior and Their Long-Term Predictive Capability." Nuc. and Chem. Waste Management $2: 307-313$.

Dagan, G. 1982. "Stochastic Modeling of Groundwater Flow by Unconditional and Conditional Probabilities, 2. The Solute Transport." Water Resources Res., $18(4): 835-848$.

Davis, L. A., and S. P. Neuman. 1983. Documentation and User's Guide: UNSAT2 - Variably Saturated Flow Mode1. NUREG/CR-3390, WWL/TM-1791-1, U.S. Nuclear Regulatory Commission, Washington, D.C.

Duguid, J. 0., and M. Reeves. 1976. Material Transport Through Porous Media: A Finite-Element Galerkin Model. OR $\overline{N L}-4928$, Oak Ridge National Laboratory, Oak Ridge, Tennessee.

Freeze, R. A., and J. A. Cherry. 1979. Groundwater. Prentice-Ha11, Englewood Cliffs, New Jersey.

General Electric Company. 1984. Chart of the Nuclides. 13th ed. General Electric Company, San Jose, California.

GTC (Geologic Testing Consultants). 1985. Shallow Disposal of Radioactive Waste: Survey of Models. INF 0-0149. Prepared for the Atomic Energy Control Board by Geologic Testing Consultants, Ottawa, Canada.

Gray, W. G. 1983. "General Conservation Equations for Multi-Phase Systems: 4. Constitutive Theory Including Phase Change." Adv. Water Resources. 6:130140 .

Grisak, G. E., and J. F. Pickens. 1981. An Analytical Solution for Solute Transport Through Fractured Media with Matrix Diffusion." J. of Hydrology. $52(1 / 2): 47-57$. 
Grisak, G. E., and J. F. Pickens. 1981. An Analytical Solution for Solute Transport Through Fractured Media with Matrix Diffusion." J. of Hydrology. $52(1 / 2): 47-57$.

Grisak, G. E., and J.F. Pickens. 1980. "Solute Transport Through Fractured Media: Volume I. The Effect of Matrix Diffusion." Water Resour. Res. $16: 719-730$.

Gupta, S. K., C. T. Kincaid, P. R. Meyer, C. A. Newbill and C. R. Cole. 1982. A Multidimensional Finite Element Code for the Analys is of Coupled Fluid, Energy, and Solute Transport (CFEST). PNL-4260, Pacific Northwest Laboratory, Richland, Washington.

Hassanizadeh, M., and W. G. Gray. 1980. "General Conservation Equations for MultiPhase Systems: 3. Constitutive Theory for Porous Media Flow." Adv. Water Resources. 3:25-40.

Kincaid, C. T., J. R., Morrey and J. E. Rogers. 1984. Geohydrochemical Modelis. for Solute Migration, Volume 1 ; Process Description and Computer Code Selection. EPRI EA-3417 Vo1. 1. Electric Power Research Institute,

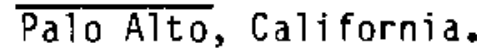

Kincaid, C. T., J. R. Morrey, S. B. Yabusaki, A. R. Felmy and J. E. Rogers. 1984. Geohydrochemical Models for Solute Migration, Volume 2; Preliminary Evaluation of Selected Computer Codes. EA-3417, Vol. 2. ETectric Power Research Institute, Palo Alto, California.

Kobayashi, H. and B. E. Rittmann. 1982. "Microbial Removal of Hazardous Organic Compounds." Environ. Sci. Technol. 16:170A-183A.

Konikow, L.F., and J. D. Bredehoeft. 1978 Techniques of Water Resources Investigations of the United States Geological Survey: Computer Model of Two-Dimensional Solute Transport and Dispersion in Ground Water. U.S. Geologic Survey, Reston, Virginia.

Matthess, G. 1984. "Unsaturated Zone Pollution by Metals." In Pollutants in Porous Media: The Unsaturated Zone Between Soil Surface and Groundwater, ed. B. Yaron et at., Springer-Vertag, New York.

McKay, M. D., W. J. Conover and R. J. Beckman. 1979. "A Comparison of Three Methods for Selecting Values of Input Variables in the Analysis of Output from a Computer." Technometrics 21:239-245.

Neuman, S. P., R. A. Feddes and E. Bresler. 1974. Finite Element Simulation of Flow in Saturated - Unsaturated Soils Considering Water Uptake by Plants. Technion, Israet Institute of Technology, Research and Development Foundation Ltd, Israel.

Oster, C. A. 1982. Review of Ground-Water Flow and Transport Models in the Unsaturated Zone. NUREG/CR-2917, U.S. Nuc lear Regutatory Commission, Washington, D.C. 
Pratt, P. F., and W. A. Jury. 1984. "Pollution of the Unsaturated Zone with Nitrate." In Pollutants in Porous Media: The Unsaturated Zone Between Soil Surface and Groundwater, ed. B. Yaron et al., Springer-Verlag, New York.

Schville, F. 1984. "Migration of Organic Fluid Immiscible with Water in the Unsaturated Zone." In Pollutants in Porous Media: The Unsaturated Zone Between Soil Surface and Groundwater, ed. B. Yaron et al., Springer-Verlag, New York.

(Sciences Applications, Inc.) 1981. Tabulation of Waste Isolation Computer Models. Office of Nuclear Waste Isolation, Battelle Project Management Division, Columbus, Ohio.

Simmons, C. S., and C.R. Cole. 1985a. Guidelines for Selecting Codes for Ground-Water Transport Modeling of Low-Level Waste Burial Sites, Executive Summary. PNL-4980, Pacific Northwest Laboratory, Richland, Washington.

Simmons, C. S., and C.R. Cole. 1985b. Guidelines for Selecting Codes for Ground-Water Transport Modeling of Low-Level Waste Burial Sites, Volume I Guidelines Approach. PNL-4980, Vol. 1, Pacific Northwest Laboratory, Richland, Washington.

Tang, D. H., E. 0. Frind and E. A. Sudicky. 1981. "Contaminant Transport in Fractured Porous Media: Analytical Solution for a Single Fracture." Water Resour. Res. $17: 555-564$.

Thomas, S. D., B. Ross and J. W. Mercer. 1982. A Summary of Repository Siting Models. NUREG/CR-2782, U.S. Nuclear Regulatory Commission, Washington, D.C.

Torak, L. J. 1982. Modifications and Corrections to the Finite-Difference Model for Simulation of Three-Dimensional Ground-Water Flow. Open-File Report 82-4025, U.S. Geological Survey, Reston, Virginia.

Tracy, J. V. 1982. Users Gujde and Documentation for Adsorption and Decay Modifications to the USGS Solute Transport Model. NUREG/CR-2502, U.S. Nuclear Regulatory Commission, Washington, D.C.

Travis, C. C., and E. L. Etnier. 1981. "A Survey of Sorption Relationships for Reactive Solutes in Soil." J. Environ. Qual. 10(1):8-17.

Trescott, P.C. 1975. Documentation of Finite-Difference Model for Simulation of Three-Dimensional Ground-Water Flow. Open-File Report 75-438, U.S. Geotogical Survey, Reston, Virginia.

Trescott, P. C., and S. P. Larson. 1976. Documentation of Finite-Difference Model for Simulation of Three-Dimensional Ground-Water Flow. Open-file Report 76-591, U.S. Geological Survey, Reston, Virginia. 
Van Dam, J. 1967. "The Migration of Hydrocarbons in a Water Bearing Stratum." In The Joint Problems of the $0 i l$ and Water Industries. Ed. P. Hepple, Institute of Petroleum, London.

Van der Heijde, P. K. M., and P. Srinvasen. 1984. "Summary Listing of Hydrochemical Models for Main-Frame and Mini-Computers." In IGWMC MARS Data Base. International Ground Water Modeling Center, Holcomb Research Institute, Butter University, Indianapolis, Indiana.

Van der Heijde, P. K. M., Y. Bachmat, J. Bredehoeft, B. Andrews, P. Holtz and S. Sebastion. 1985. "Groundwater Management: The Use of Numerical Mode 1s." In Water Resource Monograph No.5. American Geophysical Union, Washington, D.C.

Yaron, B., G. Dagan and J. Goldshmid, eds. 1984. Pollutants in Porous Media: The Unsaturated Zone Between Soil Surface and Groundwater. SpringerVerlag, New York.

Yeh, G. T., and D. S. Ward. 1981. A FEMWASTE Finite-Element Model of Waste Transport Through Saturated-Unsaturated Porous Media. ORNL-5601, Oak Ridge Nationa Laboratory, Oak Ridge, Tennessee. 


\section{DISTRIBUTION}

No. of

Copies

OFFSITE

30 O0E Technical Information Center

\section{ONSITE}

6 DOE Richland 0perations Office

P. K. Clark

D. R. Elle

P. J. Krupin

M. J. Plahuta

J. J. Sutey

G. R. Yesberger

11 Rockwe 11 Hanford Operations

M. R. Adams

C. Defigh-Price

C. J. Geier

V. W. Hall

A. G. Law

D. E. McKenney

C. C. Meinhardt

S. L. Phillips

L. L. Powers

J. F. Relyea

R. C. Routson
54 Pacific Northwest Laboratory

W. J. Bair

M. P. Bergeron

J. B. Brown, Jr.

J. W. Cary

C. R. Cole

P. A. Eddy

C. E. Elderkin

M. G. Foley

M. D. Freshley

G. W. Gee

M. J. Graham

H. A. Haerer

J. M. Hales

P. C. Hays

T. L. Jones

W. E. Kennedy, Jr.

C. T. Kincaid (10)

T. J. Mclaughlin

P. J. Mitchell (10)

D. A. Myers

R. W. Neison

L. S. Prater

U. R. Raymond

A. E. Reisenauer

C. S. Simmons

J. A. Stottlemyre

G. P. Streile

$G$. Whelan

R. E. Wildung

Publishing Coordination (2)

Technical Information (5) 
